Applied and

NISTIR 5164

Computational

Mathematics

Division

Computing and Applied Mathematics Laboratory

\title{
Bibliographic Notes on Voronoi Diagrams
}

\author{
J. Bernal
}

April 1993

\author{
Technology Administration \\ U.S. DEPARTMENT OF COMMERCE \\ National Institute of Standards and Technology \\ Gaithersburg, MD 20899
}

QC

100

.056

\#5164

1993 



\section{Bibliographic Notes on Voronoi Diagrams}

\section{J. Bernal}

U.S. DEPARTMENT OF COMMERCE

Technology Administration

National Institute of Standards

and Technology

Applied and Computational Mathematics Division Computing and Applied Mathematics Laboratory

Gaithersburg, MD 20899

April 1993

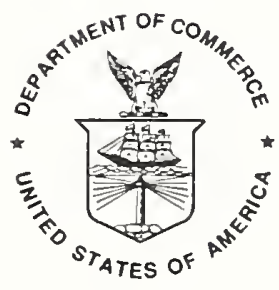

U.S. DEPARTMENT OF COMMERCE

Ronald H. Brown, Secretary

NATIONAL INSTITUTE OF STANDARDS

AND TECHNOLOGY

Raymond G. Kammer, Acting Director 



\title{
Bibliographic notes on Voronoi diagrams
}

\author{
Javier Bernal \\ National Institute of Standards and Technology, Gaithersburg, MD 20899
}

\begin{abstract}
This paper presents a comprehensive annotated bibliography on various theoretical and algorithmic aspects of Voronoi diagrams and related diagrams. Bibliographic notes on the relationship between Voronoi diagrams and solutions to the Euclidean traveling salesman problem are also presented.
\end{abstract}

Key words. Algorithm, arrangement, complexity, convex hull, Delaunay triangulation, power diagram, traveling salesman problem, Voronoi diagram

AMS (MOS) subject classifications. 68U05 


\section{Contents}

1. Initial papers

2. Sources for material on Voronoi diagrams and related topics

3. Optimality of Delaunay triangulations

4. Visibility in Delaunay triangulations

5. Convex combinations from Voronoi diagrams

6. Combinatorial properties of Voronoi diagrams

7. Algorithms for computing Voronoi diagrams and Delaunay triangulations

8. Voronoi diagrams from convex hulls or intersections of half-spaces

9. Convex hulls

10. Optimal expected-time algorithms

11. Randomized 2-dimensional incremental algorithms

12. Parallel computation of Voronoi diagrams and convex hulls

13. Robustness and stability

14. Voronoi diagram recognition

15. Constrained and conforming Delaunay triangulations

16. Voronoi diagrams for general figures

17. Voronoi diagrams for general distance measures

18. Furthest point Voronoi diagrams

19. Order $-k$ Voronoi diagrams 
20. Multiplicative and additively weighted Voronoi diagrams

21. Power diagrams and arrangements

22. Abstract Voronoi diagrams, discrete Voronoi diagrams, etc.

23. Delaunay triangulations and the Euclidean traveling salesman problem 


\section{Initial papers}

The following papers and textbook are among the first to investigate mathematical aspects of Voronoi diagrams and Delaunay triangulations.

1. G. L. Dirichlet, Über die Reduktion der positiven quadratischen Formen mit drei unbestimmten ganzen Zahlen, J. Reine Angew. Math. 40 (1850), 209-227.

2. G. Voronoi, Nouvelles applications des paramètres continus à la théorie des formes quadratiques, J. Reine Angew. Math. 134 (1908), 198-287.

3. B. Delaunay, Sur la sphère vide, Bull. Acad. Sci. USSR (VII), Classe Sci. Mat. Nat. (1934), 793-800.

4. C. A. Rogers, Packing and Covering, Cambridge University Press, Cambridge, England (1964).

\section{Sources for material on Voronoi diagrams and related topics}

The following papers present surveys about Voronoi diagrams and related topics.

1. D. T. Lee and F. P. Preparata, Computational Geometry - A survey, IEEE Transactions on Computers C-33 (1984), 1072-1101.

2. L. De Floriani and E. Puppo, A survey of constrained Delaunay triangulation algorithms for surface representation, Issues on Machine Vision, G. G. Pieroni (Ed.), Springer-Verlag, New York (1989), 95-104.

3. F. Aurenhammer, Voronoi diagrams - A survey of a fundamental geometric data structure, ACM Computing Surveys 23 (1991), 345-405.

4. S. Fortune, Voronoi diagrams and Delaunay triangulations, Computing in Euclidean Geometry, D.-Z. Du and F. K. Hwang (Ed.), World Scientific (1992), 193-233.

5. S. Fortune, Computational Geometry, Directions in Geometric Computing, R. Martin (Ed.), Information Geometers, to appear (1993).

The following textbooks are sources for material on Voronoi diagrams and related topics.

1. F. P. Preparata and M. I. Shamos, Computational Geometry - An Introduction, Springer-Verlag, New York (1985). 
2. H. Edelsbrunner, Algorithms in Combinatorial Geometry, Springer-Verlag, New York (1987).

\section{Optimality of Delaunay triangulations}

In the first of the following two papers planar triangulations that are locally equiangular are suggested for interpolation work, and a diagonal-flipping algorithm for computing them from arbitrary triangulations based on this criterion is proposed. In the second paper it is proven that given a set of points in the plane, a triangulation for the set is Delaunay if and only if it is locally equiangular. Essentially, a triangulation is locally equiangular if given any two triangles in the triangulation that share a common edge and whose union is a strictly convex quadrilateral, then replacement of the common edge by the alternative diagonal of the quadrilateral does not increase the minimum of the six angles in the two triangles making up the quadrilateral.

1. C. L. Lawson, Generation of a triangular grid with applications to contour plotting, TM 299, California Inst. Tech. Jet Propulsion Lab (1972).

2. R. Sibson, Locally equiangular triangulations, The Comput. J. 21 (1978), 243-245.

In the first of the following papers the local optimality principle is proven for triangulations in the plane. Essentially, this principle says that if a triangulation satisfies the empty circle criterion on a local basis then it satisfies it on a global basis. It is also proven in this paper that a triangulation satisfying the empty circle criterion on a global basis is Delaunay. In the third paper these results are generalized to higher dimensions. These results are useful in the development of algorithms for computing unconstrained and constrained Delaunay triangulations. Finally, in the first two papers it is proven that a triangulation for a set of points in the plane is Delaunay if and only if among all triangulations for the set it has the lexicographically largest increasing sequence of interior minimum angles of triangles.

1. C. L. Lawson, Software for $C^{1}$ surface interpolation, Mathematical Software III, J. R. Rice (Ed.), Academic Press, New York (1977), 161-194.

2. D. T. Lee, Proximity and reachability in the plane, Ph. D. thesis, Tech. Rep. R-831, Coordinated Sci. Lab., Univ. of Illinois (1978).

3. C. L. Lawson, Properties of $n$-dimensional triangulations, Computer Aided Geometric Design 3 (1986), 231-246. 
In the following textbook it is proven that given a triangulation for a set of points in the plane, if the triangulation is globally equiangular then it is Delaunay. Essentially, a triangulation is globally equiangular if among all triangulations for the set it has the lexicographically largest increasing sequence of interior angles of triangles.

1. H. Edelsbrunner, Algorithms in Combinatorial Geometry, Springer-Verlag, New York (1987).

It follows that in the absence of degeneracies the concepts of Delaunay, locally equiangular, and globally equiangular are equivalent. In the following paper it is shown how to obtain a Delaunay triangulation that is globally equiangular in the presence of degeneracies.

1. D. Mount and A. Saalfeld, Globally-equiangular triangulation of co-circular points in $O(n \log n)$ time, Proc. $4^{\text {th }}$ Ann. ACM Symp. on Computational Geometry (1988), 143-152.

In the following paper it is proven that among all triangulations for a set of points in the plane a Delaunay triangulation minimizes the largest of the circumscribed circles of the triangles. It is also shown in this paper that given a convex quadratic surface and a set of points in the plane, a triangulation for the points can be obtained such that a linear interpolation of the convex quadratic surface over each triangle in the triangulation minimizes the maximum error in any triangle among all triangulations for the points. It is shown in this paper that by scaling the points according to the coefficients of the function representation of the convex quadratic surface, this triangulation is induced by a Delaunay triangulation of the scaled points.

1. E. F. D'Azevedo and R. B. Simpson, On optimal interpolation triangle incidences, SIAM J. Sci. Stat. Comput. 10 (1989), 1063-1075.

Given an arbitrary finite set of points in the plane and function values assigned to each point, let $T$ be a triangulation for the points, and for each triangle $t$ in $T$ let $|t|$ denote the area of $t$ and $a_{t}, b_{t}, c_{t}$ the numbers for which $z=a_{t} x+b_{t} y+c_{t}$ is the equation of the plane that at each vertex of $t$ has as $z$-coordinate the function value assigned to the vertex. In the following paper it is proven that if $T$ is Delaunay then the Sobolev seminorm

$$
\sum_{t \in T}|t|\left(a_{t}^{2}+b_{t}^{2}\right)
$$

is minimized. Since this seminorm is a measurement of the roughness of the piecewise linear interpolation surface obtained by restricting each plane of the form above to its defining triangle, it is said that the Delaunay triangulation is a minimal roughness triangulation. 
1. S. Rippa, Minimal roughness property of the Delaunay triangulation, Comput. Aided Geom. Design 7 (1990), 489-497.

In the following paper it is proven that the maximum min-containment radius of a Delaunay triangulation for a set of points in $d$-space is less than the maximum min-containment radius of any other triangulation for the set. Essentially, the min-containment radius of a $d$-dimensional triangle is the radius of the smallest $d$-dimensional sphere containing the triangle.

1. V. T. Rajan, Optimality of the Delaunay triangulation in $R^{d}$, Proc. $7^{\text {th }}$ Ann. ACM Symp. on Computational Geometry (1991), 357-372.

A triangulation for a set of points in the plane is said to be optimal if it minimizes the total Euclidean edge length over all triangulations for the set. In the first of the following papers it is proven that Delaunay triangulations are not optimal. In the second paper it is shown that Delaunay triangulations are not approximately optimal. In the third paper it is shown that Delaunay triangulations can be asymptotically as long as any arbitrary triangulation. Finally, in the fourth paper it is shown that Delaunay triangulations are close to optimal on the average.

1. E. L. Lloyd, On triangulations of a set of points in the plane, Proc. $18^{\text {th }}$ Ann. IEEE Conference on the Foundations of Computer Science (1977), 228-240.

2. G. K. Manacher and A. L. Zobrist, Neither the greedy nor the Delaunay triangulation of a planar point set approximates the optimal triangulation, Inform. Process. Lett. 9 (1979), 31-34.

3. D. G. Kirkpatrick, A note on Delaunay and optimal triangulations, Inform. Process. Lett. 10 (1980), 127-128.

4. A. Lingas, The greedy and Delaunay triangulations are not bad in the average case, Inform. Process. Lett. 22 (1986), 25-31.

\section{Visibility in Delaunay triangulations}

In the first of the following papers it is shown that given a Delaunay triangulation for a set of sites in the plane, for each site there exists a partial ordering of the triangles that is consistent with the order in which the triangles are visible from the site. In the second paper it is shown that given a Delaunay triangulation for a set of sites in $d$-dimensional space, for each point in the space this partial ordering exists. 
1. L. De Floriani, B. Falcidieno, G. Nagy, and C. Pienovi, On sorting triangles in a Delaunay tessellation, Algorithmica 6 (1991), 522-532.

2. H. Edelsbrunner, An acyclicity theorem for cell complexes in $d$ dimension, Proc. $5^{\text {th }}$ Ann. ACM Symp. on Computational Geometry (1989), 145-151.

\section{Convex combinations from Voronoi diagrams}

In the first of the following papers it is shown that given a set of points $S$ in $d$-dimensional space, every point $p$ in $S$ whose Voronoi cell in the Voronoi diagram for $S$ is bounded can be expressed as a convex combination of the other points in $S$, in such a way that given $q$ in $S, q \neq p$, the coefficient that corresponds to $q$ in this convex combination is the percentage of the Voronoi cell of $p$ closer to $q$ than to any other point in $S \backslash\{p, q\}$. In the second paper it is shown how these combinations can be used to compute a Gale transform for $S$.

1. R. Sibson, A vector identity for the Dirichlet tessellation, Math. Proc. Camb. Phil. Soc. 87 (1980), 151-155.

2. F. Aurenhammer, A relationship between Gale transforms and Voronoi diagrams, Discrete Applied Mathematics 28 (1990), 83-91.

\section{Combinatorial properties of Voronoi diagrams}

In the following papers expectations of various random variables are derived concerning Voronoi diagrams and Delaunay triangulations for stationary Poisson point processes in 2and 3-dimensional spaces, such as the expected number of faces of various dimensions of any Voronoi region.

1. J. L. Meijering, Interface area, edge length, and number of vertices in crystal aggregates with random nucleation, Philips Res. Rep. 8 (1953), 270-290.

2. E. N. Gilbert, Random subdivisions of space into crystals, Ann. Math. Statist. 33 (1962), 958-972.

3. R. E. Miles, On the homogeneous planar Poisson point process, Mathematical Biosciences 6 (1970), 85-127.

4. R. E. Miles, The random division of space, Proc. Symp. on Statistical and Probabilistic Problems in Metallurgy, August 1971, Suppl. Adv. Appl. Prob. (1972), 243-266. 
In the following paper expectations of various random variables are derived concerning the portion within a cube of side $n^{1 / d}$ of the Delaunay triangulation for an infinite random point set in $d$-dimensional space.

1. M. Bern, D. Eppstein, and F. Yao, The expected extremes in a Delaunay triangulation, International J. of Computational Geometry \& Applications 1 (1991), 79-91.

In the following papers examples are given that show that the complexity of the Voronoi diagram for a set of $n$ points in 3-dimensional space is $O\left(n^{2}\right)$.

1. A. K. Dewdney and J. K. Vranch, A convex partition of $R^{3}$ with applications to Crum's problem and Knuth's post-office problem, Utilitas Mathematica 12 (1977), 193-199.

2. F. P. Preparata, Steps into computational geometry, Tech. Rep., Coordinated Science Laboratory, University of Illinois (1977).

The complexity of Voronoi diagrams in $d$-dimensional space is addressed in the following papers. In the second and third papers an exact upper bound is derived for the number of $i$-dimensional faces of Voronoi diagrams for $n$ points in $d$-dimensional space, for each $i$, $i=0, \ldots, d-1$.

1. V. Klee, On the complexity of $d$-dimensional Voronoi diagrams, Archiv. Math. 34 (1980), 75-80.

2. I. Paschinger, Konvexe Polytope und Dirichletsche Zellenkomplexe, Ph. D. dissertation, Inst. Math., Univ. Salzburg, Austria (1982).

3. R. Seidel, The complexity of Voronoi diagrams in higher dimensions, Proc. $20^{\text {th }}$ Ann. Allerton Conference on Communication, Control, and Computing (1982), 94-95.

\section{Algorithms for computing Voronoi diagrams and Delaunay triangulations}

The following papers present $O(n \log n)$ worst-case optimal divide-and-conquer algorithms for computing the Voronoi diagram or a Delaunay triangulation for a set of $n$ points in the plane.

1. M. I. Shamos and D. Hoey, Closest-point problems, Proc. $16^{\text {th }}$ Ann. IEEE Symp. on Foundations of Computer Science (1975), 151-162.

2. D. T. Lee, Proximity and reachability in the plane, Ph. D. thesis, Tech. Rep. R-831, Coordinated Sci. Lab., Univ. of Illinois (1978). 
3. M. I. Shamos, Computational geometry, Ph. D. thesis, Dept. of Comput. Sci., Yale Univ. (1978).

4. D. T. Lee and B. J. Schachter, Two algorithms for constructing a Delaunay triangulation, Int. J. Comput. Inf. Sci. 9 (1980), 219-242.

5. L. Guibas and J. Stolfi, Primitives for the manipulation of general subdivisions and the computation of Voronoi diagrams, ACM Transactions on Graphics 4 (1985), 74-123.

6. R. A. Dwyer, A faster divide-and-conquer algorithm for constructing Delaunay triangulations, Algorithmica 2 (1987), 137-151.

The following papers present incremental algorithms for computing 2-dimensional Voronoi diagrams or Delaunay triangulations.

1. D. H. McLain, Two dimensional interpolation from random data, The Comput. J. 19 (1976), 178-181.

2. C. L. Lawson, Software for $C^{1}$ surface interpolation, Mathematical Software III, J. R. Rice (Ed.), Academic Press, New York (1977), 161-194.

3. H. Akima, A method of bivariate interpolation and smooth surface fitting for irregularly distributed data points, ACM Trans. Math. Softw. 4 (1978), 148-159, 160-164.

4. P. J. Green and R. Sibson, Computing Dirichlet tessellations in the plane, The Comput. J. 21 (1978), 168-173.

5. K. E. Brassel and D. Reif, A procedure to generate Thiessen polygons, Geographical Analysis 11 (1979), 289-303.

6. D. T. Lee and B. J. Schachter, Two algorithms for constructing a Delaunay triangulation, Int. J. Comput. Inf. Sci. 9 (1980), 219-242.

7. M. J. McCullagh and C. G. Ross, Delaunay triangulation of a random data set for isarithmic mapping, The Cartographic J. 17 (1980), 93-99.

8. A. K. Cline and R. L. Renka, A storage-efficient method for construction of a Thiessen triangulation, Rocky Mountain J. of Mathematics 14 (1984), 119-139.

9. L. Guibas and J. Stolfi, Primitives for the manipulation of general subdivisions and the computation of Voronoi diagrams, ACM Transactions on Graphics 4 (1985), 74-123. 
10. S. W. Sloan, A fast algorithm for constructing Delaunay triangulations in the plane, Adv. Eng. Software 9 (1987), 34-55.

The following paper presents an $O(n \log n)$ plane-sweep algorithm for computing the Voronoi diagram for a set of $n$ points in the plane.

1. S. Fortune, A sweepline algorithm for Voronoi diagrams, Algorithmica 2 (1987), 153174.

In the following paper it is shown how to maintain dynamically a Voronoi diagram for a set of $n$ points in the plane in $O(n)$ time per insertion and $O(n \log n / k(n))$ time per deletion using $O(n \log (k(n)))$ storage, where $k(n)=O(\log n)$.

1. I. G. Gowda, D. G. Kirkpatrick, D. T. Lee, and A. Naamad, Dynamic Voronoi diagrams, IEEE Transactions on Information Theory IT-29 (1983), 724-731.

The following paper presents an $O(n \log n)$ procedure for computing a globally equiangular triangulation for a set of $n$ co-circular points in the plane.

1. D. Mount and A. Saalfeld, Globally-equiangular triangulation of co-circular points in $O(n \log n)$ time, Proc. $4^{\text {th }}$ Ann. ACM Symp. on Computational Geometry (1988), 143-152.

The following papers present algorithms for computing the Voronoi diagram or a Delaunay triangulation for a set of $n$ points in 3-dimensional space. All three algorithms have worst case time complexity $O\left(n^{4}\right)$. The algorithm in the first paper first computes the facets of each Voronoi region and from them the edges and vertices of the region. In practice the complexity for this algorithm is actually $O\left(n^{2} \log n\right)$. The algorithm in the second paper computes the vertices of each Voronoi region and by identifying the sites that define each vertex computes the edges and facets of the region. Finally, the algorithm in the third paper computes the Voronoi region for each site by computing all Delaunay tetrahedra that have the site as a common vertex.

1. W. Browstow, J.-P. Dussault, and B. L. Fox, Construction of Voronoi polyhedra, J. Comput. Phys. 29 (1978), 81-97.

2. J. L. Finney, A procedure for the construction of Voronoi polyhedra, J. Comput. Phys. 32 (1979), 137-143.

3. M. Tanemura, T. Ogawa, and N. Ogita, A new algorithm for three-dimensional Voronoi tessellation, J. Comput. Phys. 51 (1983), 191-207. 
The following papers present algorithms for computing the Voronoi diagram or a Delaunay triangulation for a set of $n$ points in $d$-dimensional space. The algorithms in the first two papers are insertion algorithms for computing, respectively, the Voronoi diagram and the Delaunay triangulation for the set. Inductively, with these algorithms the $k^{\text {th }}$ point in the set is inserted into the Voronoi diagram or Delaunay triangulation for the first $k-1$ points and the Voronoi diagram or Delaunay triangulation for the $k$ points is then obtained. In these papers it is claimed that on the average the complexity of the algorithms is $O\left(n^{1+1 / d}\right)$ and $O\left(n^{(2 d-1) / d}\right)$, respectively, for a random set. Finally, the third paper presents two algorithms. The first algorithm employs a breadth first search for computing the Voronoi diagram for the set of points in worst-case time $O\left(d n^{[d / 2\rceil+1}\right)+O\left(d^{3} n^{\lceil d / 2\rceil} \log n\right)$ in the absence of degeneracies. The second algorithm employs linear programming techniques for computing a Delaunay triangulation for the set.

1. A. Bowyer, Computing Dirichlet tessellations, The Comput. J. 24 (1981), 162-166.

2. D. F. Watson, Computing the $n$-dimensional Delaunay tessellation with application to Voronoi polytopes, The Comput. J. 24 (1981), 167-172.

3. D. Avis and B. K. Bhattacharya, Algorithms for computing $d$-dimensional Voronoi diagrams and their duals, Adv. Comput. Res. 1 (1983), 159-180.

The following papers present an incremental triangle-flipping algorithm for computing a Delaunay triangulation for a set of $n$ points in 3-dimensional space in at most $O\left(n^{2}\right)$ time. Results for random problems are presented in these papers from which it can be seen that the empirical time complexity of the algorithm is $O\left(n^{4 / 3}\right)$ or $O\left(n(\log n)^{2}\right)$ for sets of random points. In the first paper it is also shown that improving an arbitrary 3 -dimensional triangulation by triangle-flipping does not necessarily terminate in a Delaunay triangulation.

1. B. Joe, Three-dimensional triangulations from local transformations, SIAM J. Sci. Stat. Comput. 10 (1989), 718-741.

2. B. Joe, Construction of three-dimensional Delaunay triangulations using local transformations, Computer Aided Geometric Design 8 (1991), 123-142.

The following paper presents an incremental triangle-flipping algorithm for computing a Delaunay triangulation for a set of $n$ points in $d$-space in at most $O\left(n^{\lceil(d+1) / 2\rceil} \log n+n^{\lceil d / 2\rceil+1}\right)$ time.

1. V. T. Rajan, Optimality of the Delaunay triangulation in $R^{d}$, Proc. $7^{\text {th }}$ Ann. ACM Symp. on Computational Geometry (1991), 357-372. 
The following paper presents an incremental triangle-flipping algorithm for computing a Delaunay triangulation for a set of $n$ points in $d$-space in expected $O\left(n \log n+n^{\lceil d / 2\rceil}\right)$ time if the points are added in a random sequence.

1. H. Edelsbrunner and N. R. Shah, Incremental topological flipping works for regular triangulations, Proc. $8^{\text {th }}$ Ann. ACM Symp. on Computational Geometry (1992), 43-52.

The following papers present data structures for storing Voronoi diagrams and Delaunay triangulations that are convenient for the implementation of divide-and-conquer and incremental algorithms for computing Voronoi diagrams and Delaunay triangulations. The first and second papers deal with the $2-$ and 3 -dimensional cases, respectively. The last two papers deal with the $d$-dimensional case.

1. L. Guibas and J. Stolfi, Primitives for the manipulation of general subdivisions and the computation of Voronoi diagrams, ACM Transactions on Graphics 4 (1985), 74-123.

2. D. P. Dobkin and M. J. Laszlo, Primitives for the manipulation of three-dimensional subdivisions, Algorithmica 4 (1989), 3-32.

3. E. Brisson, Representing geometric structures in $d$ dimensions: topology and order, Proc. $5^{\text {th }}$ Ann. ACM Symp. on Computational Geometry (1989), 218-227.

4. P. Lienhardt, Subdivisions of $n$-dimensional spaces and $n$-dimensional generalized maps, Proc. $5^{\text {th }}$ Ann. ACM Symp. on Computational Geometry (1989), 228-236.

The following papers present perturbation schemes of input data for geometric programs that eliminate degeneracies. Softwares for computing Voronoi diagrams and convex hulls in $d$-dimensional space are examples of geometric programs for which it is practical to use these techniques.

1. C.-K. Yap, Symbolic treatment of geometric degeneracies, J. Symbolic Computation 10 (1990), 349-370.

2. C.-K. Yap, A geometric consistency theorem for a symbolic perturbation scheme, J. of Computer and System Sciences 40 (1990), 2-18.

3. H. Edelsbrunner and E. P. Mücke, Simulation of simplicity: A technique to cope with degenerate cases in geometric algorithms, ACM Transactions on Graphics 9 (1990), 66-104. 
4. I. Emiris and J. Canny, A general approach to removing degeneracies, Proc. $32^{\text {nd }}$ Ann. IEEE Symp. on the Foundations of Computer Science (1991), 405-413.

5. I. Emiris and J. Canny, An efficient approach to removing geometric degeneracies, Proc. $8^{\text {th }}$ Ann. ACM Symp. on Computational Geometry (1992), 74-82.

\section{Voronoi diagrams from convex hulls or intersections of half-spaces}

In the following papers it is shown how to compute a $d$-dimensional Voronoi diagram by transforming the data points to $(d+1)$-space using geometric inversion, computing the convex hull of the transformed points, and then transforming the convex hull back to $d$-space.

1. K. Q. Brown, Voronoi diagrams from convex hulls, Info. Proc. Lett. 9 (1979), 223-228.

2. K. Q. Brown, Geometric transforms for fast geometric algorithms, Ph. D. thesis, Rep. CMU-CS-80-101, Dept. of Comput. Sci., Carnegie-Mellon Univ. (1980).

In the following papers it is shown how to compute $d$-dimensional Voronoi diagrams as perpendicular projections onto $d$-dimensional space of the boundaries of polyhedra that are intersections of half-spaces in $(d+1)$-dimensional space.

1. I. Paschinger, Konvexe Polytope und Dirichletsche Zellenkomplexe, Ph. D. dissertation, Math. Inst., Univ. Salzburg, Austria (1982).

2. H. Edelsbrunner and R. Seidel, Voronoi diagrams and arrangements, Discrete Comput. Geom. 1 (1986), 25-44.

3. H. Edelsbrunner, J. O'Rourke, and R. Seidel, Constructing arrangements of lines and hyperplanes with applications, SIAM J. Comput. 15 (1986), 341-363.

4. F. Aurenhammer, Power diagrams: properties, algorithms and applications, SIAM J. Comput. 16 (1987), 78-96.

The following paper presents a result for computing certain kinds of 3-dimensional convex hulls that implies a linear algorithm for deleting a site from a Voronoi diagram in the plane and updating the diagram. The same result implies a linear algorithm for computing the Voronoi diagram for the vertices of a convex polygon.

1. A. Aggarwal, L. J. Guibas, J. Saxe, and P. W. Shor, A linear-time algorithm for computing the Voronoi diagram of a convex polygon, Discrete Comput. Geom. 4 (1989), 591-604. 


\section{Convex hulls}

Convex hulls are closely related to Voronoi diagrams, and algorithms for computing convex hulls can be used for computing Voronoi diagrams and Voronoi cells. The following papers present algorithms for computing planar convex hulls. With $n$ as the size of the input and $A$ as the size of the output, the first and fourth papers present algorithms with complexity $n \log n$, the second and third papers present algorithms with complexity $n A$, and the last paper presents an algorithm with complexity $n \log A$.

1. R. L. Graham, An efficient algorithm for determining the convex hull of a finite planar set, Inform. Proc. Lett. 1 (1972), 132-133.

2. R. A. Jarvis, On the identification of the convex hull of a finite set of points in the plane, Inform. Proc. Lett. 2 (1973), 18-21.

3. W. F. Eddy, A new convex hull algorithm for planar sets, ACM Trans. Math. Software 3 (1977), 398-403, 411-412.

4. J. L. Bentley and M. I. Shamos, Divide and conquer for linear expected time, Inform. Process. Lett. 7 (1978), 87-91.

5. D. G. Kirkpatrick and R. Seidel, The ultimate planar convex hull algorithm?, SIAM J. on Computing 15 (1986), 287-299.

The following paper presents divide-and-conquer algorithms of $O(n \log n)$ worst-case optimal complexity for computing the convex hulls for sets of $n$ points in two and three dimensions.

1. F. P. Preparata and S. J. Hong, Convex hulls of finite sets of points in two and three dimensions, Comm. ACM 20 (1977), 87-93.

In the following paper it is shown how to compute in $O(n \log n)$ worst-case optimal complexity the steady-state convex hull for a set of $n$ points in $k$-motion in $d$-dimensional space, $d \leq 3$. A point is in $k$-motion if its position in the space can be described in terms of a polynomial with degree $\leq k$. Steady-state refers to conditions at time $t=\infty$.

1. M. J. Atallah, Dynamic computational geometry, Proc. $24^{\text {th }}$ Ann. IEEE Symp. on Foundations of Computer Science (1983), 92-99.

The following papers and textbooks present algorithms for computing convex hulls in higher dimensions. In the third paper it is shown that the worst-case complexity of the gift-wrapping 
algorithm in the first paper is $O\left(d n^{[(d-1) / 2]+1}\right)+O\left(d^{3} n^{\lceil(d-1) / 2\rceil} \log n\right)$ for computing the convex hull for $n$ points in $d$-dimensional space. In the second paper an algorithm is presented which is optimal for even $d$, for computing in $O\left(n \log n+n^{[d / 2\rceil}\right)$ time and $O\left(n+n^{\lfloor d / 2\rfloor}\right)$ space the convex hull for $n$ points in $d$-dimensional space, $d \geq 4$. The fifth paper or textbook presents an on-line beneath-beyond algorithm for computing in $O\left(n^{\lfloor d / 2\rfloor+1}\right)$ time the convex hull for $n$ points in $d$-dimensional space. The sixth paper or textbook presents an algorithm based on the same technique for computing in $O\left(n \log n+n^{\lfloor(d+1) / 2\rfloor}\right)$ time and $O\left(n^{\lfloor d / 2\rfloor}\right)$ space the convex hull for $n$ points in $d$-dimensional space. Finally, the seventh paper presents an algorithm for computing the convex hull for $n$ points in $d$-dimensional space in optimal $O\left(n^{\lfloor d / 2\rfloor}\right)$ time for any $d>3$. A consequence of this algorithm is that the Voronoi diagram for $n$ points in $d$-dimensional space can be computed in optimal $O\left(n \log n+n^{[d / 2\rceil}\right)$ time for any $d$.

1. D. R. Chand and S. S. Kapur, An algorithm for convex polytopes, J. Assoc. Comput. Mach. 17 (1970), 78-86.

2. R. Seidel, A convex hull algorithm optimal for point sets in even dimensions, M. S. thesis, Tech. Rep. 81-14, Dept. of Comput. Sci., Univ. of British Columbia (1981).

3. B. K. Bhattacharya, Worst-case analysis of a convex hull algorithm, Tech. Rep., Dept. of Comput. Sci., Simon Fraser Univ. (1982).

4. G. Swart, Finding the convex hull facet by facet, J. of Algorithms 6 (1985), 17-48.

5. F. P. Preparata and M. I. Shamos, Computational Geometry - An Introduction, Springer-Verlag, New York (1985).

6. H. Edelsbrunner, Algorithms in Combinatorial Geometry, Springer-Verlag, New York (1987).

7. B. Chazelle, An optimal convex hull algorithm and new results on cuttings, Proc. $32^{\text {nd }}$ Ann. Symp. on Foundations of Computer Science (1991), 29-38.

The size of the convex hull of a set of $n$ points in $d$-space can be as large as $O\left(n^{\lfloor d / 2\rfloor}\right)$. However, since at times it is smaller than this number, it may be desirable to use an algorithm for computing convex hulls in $d$ dimensions whose complexity depends on the size of the output. The following paper presents one such algorithm. For fixed $d$ it has worst case time complexity $O\left(n^{2}+F \log n\right)$, where $n$ is the size of the input and $F$ is the size of the output. 
1. R. Seidel, Constructing higher-dimensional convex hulls at logarithmic cost per face, Proc. $18^{\text {th }}$ Ann. ACM Symp. on Theory of Computing (1986), 404-413.

The following papers present a randomized incremental algorithm for computing the convex hull for a nondegenerate set of $n$ points in $d$-dimensional space that requires $O(n \log n)$ expected time for $d=3$, and $O\left(n^{\lfloor d / 2\rfloor}\right)$ expected time for $d>3$. The second paper presents an algorithm that through the use of random sampling computes the convex hull for a set of $n$ points in 3-dimensional space in $O(n \log A)$ expected time, where $A$ is the size of the output.

1. K. L. Clarkson and P. W. Shor, Algorithms for diametral pairs and convex hulls that are optimal, randomized, and incremental, Proc. $4^{\text {th }}$ Ann. ACM Symp. on Computational Geometry (1988), 12-17.

2. K. L. Clarkson and P. W. Shor, Applications of random sampling in computational geometry, II, Discrete Comput. Geom. 4 (1989), 387-421.

The following paper presents a randomized incremental algorithm for computing the convex hull for a nondegenerate set of $n$ points in $d$-dimensional space, $d>3$, in $O\left(n^{\lfloor d / 2\rfloor}\right)$ expected time without having to maintain conflict graphs.

1. R. Seidel, Linear programming and convex hulls made easy, Proc. $6^{\text {th }}$ Ann. ACM Symp. on Computational Geometry (1990), 211-215.

The following paper presents an algorithm for computing in $O(n \log n)$ time the convex hull for a set of $n$ discs in the plane.

1. D. Rappaport, A convex hull algorithm for discs, and applications, Computational Geometry: Theory and Applications 1 (1992), 171-187.

\section{Optimal expected-time algorithms}

The following papers present linear expected time algorithms for computing the Voronoi diagram or a Delaunay triangulation for a set of uniformly distributed points in a square or rectangle in the plane.

1. J. L. Bentley, B. W. Weide, and A. C. Yao, Optimal expected-time algorithms for closest point problems, ACM Trans. Math. Softw. 6 (1980), 563-580.

2. A. Maus, Delaunay triangulation and the convex hull of $n$ points in expected linear time, BIT 24 (1984), 151-163. 
3. T. Ohya, M. Iri, and K. Murota, A fast Voronoi diagram algorithm with quaternary tree bucketing, Inf. Process. Lett. 18 (1984), 227-231.

4. T. Ohya, M. Iri, and K. Murota, Improvements of the incremental methods for the Voronoi diagram with computational comparison of various algorithms, J. Operations Res. Soc. Japan 27 (1984), 306-337.

5. T. Asano, M. Edahiro, H. Imai, M. Iri, and K. Murota, Practical use of bucketing techniques in computational geometry, Computational Geometry, G. T. Toussaint (Ed.), North-Holland (1985), 153-195.

The following papers present a linear expected time algorithm for computing the Voronoi diagram for a set of uniformly distributed points in a 3-dimensional cube.

1. J. Bernal, On the expected complexity of the 3-dimensional Voronoi diagram, National Institute of Standards and Technology IR 4321 (1990).

2. J. Bernal, An expected linear 3-dimensional Voronoi diagram algorithm, National Institute of Standards and Technology IR 4340 (1990).

The following paper presents a general method for determining the expected complexity of Voronoi diagrams for random point sets in $d$-dimensional space. It also presents a linear expected time algorithm for computing the Voronoi diagram for a set of uniformly distributed points in a $d$-dimensional ball.

1. R. A. Dwyer, Higher-dimensional Voronoi diagrams in linear expected time, Discrete Comput. Geom. 6 (1991), 343-367.

\section{Randomized 2-dimensional incremental algorithms}

Incremental algorithms for computing Voronoi diagrams in the plane have the advantage of being simple to implement. Even though for certain situations their complexities can be undesirably high, it has been shown that if the points in the set are introduced in random order then the Voronoi diagram or a Delaunay triangulation for the set can be computed in expected $O(n \log n)$ time and expected $O(n)$ space, where $n$ is number of points in the set. The following papers deal with the incremental randomized construction of 2-dimensional Voronoi diagrams or Delaunay triangulations. The first paper introduces a data structure called the Delaunay tree which links together in a hierarchical fashion all triangles that arise in the incremental construction of a Delaunay triangulation. This structure can be used 
to implement simple algorithms for the incremental construction of Delaunay triangulations in any dimension since it can be updated efficiently each time a new site is introduced. A data structure similar to the Delaunay tree is used to define the algorithm in the fifth paper. The second and third papers deal with the incremental randomized construction of 3-dimensional convex hulls. The algorithms in the fourth and fifth papers are based on the technique presented in the second paper. The algorithm in the fourth paper extends to a subset of the class of abstract Voronoi diagrams. Finally, in the sixth paper it is shown that using dynamic sampling a random site can be added to a Voronoi diagram or Delaunay triangulation for $n$ sites in expected $O(\log n)$ time, and deleted in expected $O(1)$ time.

1. J.-D. Boissonnat and M. Teillaud, An hierarchical representation of objects: The Delaunay tree, Proc. $2^{\text {nd }}$ Ann. ACM Symp. on Computational Geometry (1986), 260-268.

2. K. L. Clarkson and P. W. Shor, Algorithms for diametral pairs and convex hulls that are optimal, randomized, and incremental, Proc. $4^{\text {th }}$ Ann. ACM Symp. on Computational Geometry (1988), 12-17.

3. K. L. Clarkson and P. W. Shor, Applications of random sampling in computational geometry, II, Discrete Comput. Geom. 4 (1989), 387-421.

4. K. Mehlhorn, C.O'Dunlaing, and S. Meiser, On the construction of abstract Voronoi diagrams, Springer-Verlag Lecture Notes in Computer Science 415 (1990), 227-239.

5. L. J. Guibas, D. E. Knuth, and M. Sharir, Randomized incremental construction of Delaunay and Voronoi diagrams, Springer-Verlag Lecture Notes in Computer Science 443 (1990), 414-431.

6. K. Mulmuley, Randomized multidimensional search trees: dynamic sampling, Proc. $7^{\text {th }}$ Ann. ACM Symp. on Computational Geometry (1991), 121-131.

\section{Parallel computation of Voronoi diagrams and convex hulls}

The following paper presents an algorithm for the parallel computation of the convex hull for a set of $n$ points in 3-dimensional space in $O\left(\log ^{3} n\right)$ time using $O(n)$ processors.

1. A. Chow, Parallel algorithms for geometric problems, Ph. D. dissertation, Dept. of Comput. Sci., Univ. of Illinois at Urbana-Champaign (1980).

The following paper presents an algorithm for the parallel computation of the convex hull for a set of $n$ points in the plane in $O\left(\log ^{2} n\right)$ time using $n$ processors and $O((1 / \alpha) \log n)$ time using $n^{1+\alpha}$ processors, $0<\alpha \leq 1$. 
1. A. Chow, A parallel algorithm for determining convex hulls of sets of points in two dimensions, Proc. $19^{\text {th }}$ Allerton Conference on Communication, Control and Computing (1981), 214-233.

The following paper presents an algorithm for the parallel computation of the convex hull for a set of $n$ points in the plane in $O(\log n)$ time using $O(n)$ processors.

1. M. J. Atallah and M. T. Goodrich, Efficient parallel solutions to geometric problems, Proc. 1985 IEEE Intl. Conf. on Parallel Processing (1985), 411-417.

The following paper presents algorithms for the parallel computation of the Voronoi diagram for a set of $n$ points in the plane in $O\left(\log ^{2} n\right)$ time using $O(n)$ processors, and the convex hull for a set of $n$ points in 3-dimensional space in $O\left(\log ^{2} n \log ^{*} n\right)$ time using $O(n)$ processors.

1. N. Dadoun and D. G. Kirkpatrick, Parallel processing for efficient subdivision search, Proc. $3^{\text {rd }}$ Ann. ACM Symp. on Computational Geometry (1987), 205-214.

The following paper presents algorithms for the parallel computation of the convex hull and Voronoi diagram for a set of $n$ points in the plane in $O(\log n)$ and $O\left(\log ^{2} n\right)$ times, respectively, using $O(n)$ processors, and the convex hull for a set of $n$ points in 3-dimensional space in $O\left(\log ^{3} n\right)$ time using $O(n)$ processors.

1. A. Aggarwal, B. Chazelle, L. Guibas, C. Ó’Dúnlaing, and C. Yap, Parallel computational geometry, Algorithmica 3 (1988), 293-327.

The following paper presents an $O\left(\log ^{2} n\right)$ algorithm for computing the Delaunay triangulation for $n$ points in the discrete universe of grid points in the plane of the form $p=\left(p_{x}, p_{y}\right)$, $p_{x}, p_{y}$ integers, $0 \leq p_{x}, p_{y} \leq 2^{k}-1, k$ a fixed integer, using $n^{2}$ processors with word length $O(k+\log n)$.

1. O. Schwarzkopf, Parallel computation of discrete Voronoi diagrams, Springer-Verlag Lecture Notes in Computer Science 349 (1989), 193-204.

The following paper presents a randomized algorithm for the parallel computation with high probability of the convex hull for a set of $n$ points in 3-dimensional space in $O(\log n)$ time using $n$ processors.

1. J. H. Reif and S. Sen, Polling: A new randomized sampling technique for computational geometry, Proc. 21 ${ }^{\text {st }}$ Ann. ACM Symp. on Theory of Computing (1989), 394-404. 
The following paper presents two algorithms for the parallel computation of the Voronoi diagram for a set of $n$ points in the plane in $O(\log n \log \log n)$ time and $O\left(n \log ^{2} n\right)$ work, and $O\left(\log ^{2} n\right)$ time and $O(n \log n)$ work, respectively.

1. R. Cole, M. T. Goodrich, and C. Ó'Dúnlaing, Merging free trees in parallel for efficient Voronoi diagram construction, Springer-Verlag Lecture Notes in Computer Science 443 (1990), 432-445.

\section{Robustness and stability}

The following papers present methods for computing in a numerically stable manner Voronoi diagrams in the plane. The Voronoi diagrams computed in this fashion are not necessarily correct but converge to the true Voronoi diagrams as the computational precision is increased.

1. K. Sugihara and M. Iri, Geometric algorithms in finite-precision arithmetic, RMI 88-10, Faculty of Engineering, Univ. of Tokyo, Japan (1988).

2. K. Sugihara and M. Iri, Construction of the Voronoi diagram for over one million generators in single-precision arithmetic, Proc. $1^{\text {st }}$ Canad. Conf. Comput. Geom. (1989).

The following paper presents a method for computing correct Delaunay triangulations in the plane while avoiding numerical errors and nonexistent degeneracies.

1. K. Sugihara, A simple method for avoiding numerical errors and degeneracy in Voronoi diagram construction, RMI 88-14, Faculty of Engineering, Univ. of Tokyo, Japan (1988).

The following paper presents a general framework for implementing robust geometric algorithms using finite precision arithmetic. In this framework algorithms compute an exact result for a perturbed version of the input data, and return a bound on the size of the required perturbation based on the size of the rounding errors observed during the computation. In this paper special consideration is given to the implementation of basic geometric predicates of 2-dimensional geometry such as predicates for testing whether two points are distinct, whether three points are collinear, whether a point is between two other points, etc. It is also shown in this paper how these basic predicates can be combined to produce more complex algorithms.

1. L. Guibas, D. Salesin, and J. Stolf, Epsilon geometry: Building robust algorithms from imprecise computations, Proc. $5^{\text {th }}$ Ann. ACM Symp. on Computational Geometry (1989), 208-217. 
The following paper presents stable algorithms for computing 2-dimensional convex hulls and maintaining a planar triangulation if the arithmetic operations involved make relative error $\epsilon$. The first algorithm computes a convex hull that is the correct convex hull for points that have been relatively perturbed by at most $O(\epsilon)$. The second algorithm maintains a triangulation for a set of $n$ points in the plane through the operations of point location, point addition, point deletion, and diagonal flipping, in such a way that after any sequence of operations the graph structure of the triangulation and the coordinates of the input points are maintained, and the triangulation can be made planar by relatively perturbing the points by at most $O\left(n^{2} \epsilon\right)$.

1. S. Fortune, Stable maintenance of point set triangulations in two dimensions, Proc. $30^{\text {th }}$ Ann. IEEE Symp. on the Foundations of Computer Science (1989), 494-499.

In the following paper it is shown how long integer words must be in a binary computer in order to compute exact Delaunay triangulations using integer arithmetic.

1. M. Jünger, G. Reinelt, and D. Zepf, Computing correct Delaunay triangulations, Computing 47 (1991), 43-49.

The following paper presents adaptive-precision algorithms for accelerating the performance of rational arithmetic during the computation of the sign of the determinant of a matrix. Reduced running times are reported in this paper for a rational arithmetic implementation of a Delaunay triangulation algorithm into which these algorithms have been integrated.

1. M. Karasick, D. Lieber, and L. R. Nackman, Efficient Delaunay triangulation using rational arithmetic, ACM Transactions on Graphics 10 (1991), 71-91.

In the following paper it is shown that a diagonal-flipping algorithm and an incremental algorithm for computing 2-dimensional Delaunay triangulations can be implemented reliably in approximate arithmetic. The output of each algorithm is a triangulation which is an approximate Delaunay triangulation in the sense that a slight contraction of the circumcircle of each triangle contains no sites in its interior.

1. S. Fortune, Numerical stability of algorithms for 2D Delaunay triangulations, Proc. $8^{\text {th }}$ Ann. ACM Symp. on Computational Geometry (1992), 83-92.

\section{Voronoi diagram recognition}

In the following papers algorithms are given for deciding whether a simple cell complex in 
$d$-dimensional space is a Voronoi diagram for some set of points and for computing the set if it exists. Essentially, the algorithm in the first paper tests for the concurrence in each cell of rays that originate at the vertices of the cell and that are obtained by extending into the cell the edges in the complex adjacent and exterior to the cell, and by reflecting each extension in the bisector of the angle of the cell adjacent to the extension, and if these concurrences exist computes from them the set of points. Under the requirement that a cell complex be given by its incidence lattice, the algorithm in the second paper essentially computes an orthogonal dual for the cell complex if it exists and from this dual computes the set of points if it exists. The time required by the algorithm in the first paper is proportional to the number of vertices in the cell complex. The time required by the algorithm in the second paper is proportional to the number of facets. A $d$-dimensional cell complex is simple if each of its vertices is a vertex of exactly $d+1$ cells in the complex.

1. P. F. Ash and E. D. Bolker, Recognizing Dirichlet tessellations, Geometriae Dedicata 19 (1985), 175-206.

2. F. Aurenhammer, Recognising polytopical cell complexes and constructing projection polyhedra, J. Symbolic Comput. 3 (1987), 249-255.

For practical situations it is unlikely that a diagram obtained through an observation process should turn out to be an exact Voronoi diagram even if from thoretical considerations it is supposed to be one. For such a diagram, geometrical methods used for deciding whether a tessellation is Voronoi most likely would produce a negative answer. In the following paper the problem of obtaining a Voronoi diagram that approximates a given tessellation of a bounded set in the plane is considered. This problem includes the problem of computing the generators for a known Voronoi diagram. In this paper this problem is formulated as an optimization problem and an algorithm is proposed that computes a possibly locally optimal solution for it.

1. A. Suzuki and M. Iri, Approximation of a tessellation of the plane by a Voronoi diagram, J. Operations Res. Soc. Japan 29 (1986), 69-96.

\section{Constrained and conforming Delaunay triangulations}

Given a collection of vertices $V$ and edges $E$ in the plane such that the endpoints of the edges in $E$ belong to $V$ and two edges in $E$ intersect at most at endpoints, a Delaunay triangulation for $V$ constrained by $E$ is essentially a triangulation for $V$ that contains all edges in $E$ and that best approximates a Delaunay triangulation for $V$. On the other hand, 
a Voronoi diagram for $V$ contrained by $E$ can be defined in which the edges in $E$ are viewed as obstacles in the diagram and whose dual structure essentially turns out to be a Delaunay triangulation for $V$ constrained by $E$. In the following paper it is proven that a triangulation is constrained Delaunay if and only if it is constrained locally equiangular.

1. D. T. Lee and A. K. Lin, Generalized Delaunay triangulation for planar graphs, Discrete Comput. Geom. 1 (1986), 201-217.

The following papers establish the duality between a Delaunay triangulation and the Voronoi diagram for a set of points constrained by a set of line segments.

1. C. A. Wang and L. Schubert, An optimal algorithm for constructing the Delaunay triangulation of a set of line segments, Proc. $3^{\text {rd }}$ Ann. ACM Symp. on Computational Geometry (1987), 223-232.

2. R. Seidel, Constrained Delaunay triangulation and Voronoi diagrams with obstacles, Rep. 260, IIG-TU Graz, Austria (1988), 178-191.

3. A. Lingas, Voronoi diagrams with barriers and the shortest diagonal problem, Inf. Process. Lett. 32 (1989), 191-198.

The following papers present algorithms for computing a constrained Delaunay triangulation for a simple polygon. The last paper presents an $O(n \log n)$ algorithm, where $n$ is the number of vertices of the polygon.

1. B. A. Lewis and J. S. Robinson, Triangulation of planar regions with applications, The Comput. J. 21 (1978), 324-332.

2. L. De Floriani, B. Falcidieno, and C. Pienovi, Delaunay-based representation of surfaces defined over arbitrarily shaped domains, Computer Vision, Graphics, and Image Processing 32 (1985), 127-140.

3. D. T. Lee and A. K. Lin, Generalized Delaunay triangulation for planar graphs, Discrete Comput. Geom. 1 (1986), 201-217.

The following papers present algorithms for computing a Delaunay triangulation or a Voronoi diagram for a set of $n$ points constrained by a set of line segments. The second and seventh papers present $O(n \log n)$ divide-and-conquer algorithms. The sixth paper presents an $O(n \log n)$ plane-sweep algorithm. 
1. D. T. Lee and A. K. Lin, Generalized Delaunay triangulation for planar graphs, Discrete Comput. Geom. 1 (1986), 201-217.

2. C. A. Wang and L. Schubert, An optimal algorithm for constructing the Delaunay triangulation of a set of line segments, Proc. $3^{\text {rd }}$ Ann. ACM Symp. on Computational Geometry (1987), 223-232.

3. J. Bernal, On constructing Delaunay triangulations for sets constrained by line segments, National Institute of Standards and Technology Technical Note 1252 (1988).

4. J. D. Boissonnat, O. D. Faugeras, and E. Le Bras-Mehlman, Representing stereo data with the Delaunay triangulation, Proc. IEEE Intl. Conf. Robotics and Automation (1988), 24-29.

5. L. De Floriani and E. Puppo, Constrained Delaunay triangulation for multiresolution surface description, Proc. $9^{\text {th }}$ International Conference on Pattern Recognition (1988), 566-569.

6. R. Seidel, Constrained Delaunay triangulation and Voronoi diagrams with obstacles, Rep. 260, IIG-TU Graz, Austria (1988), 178-191.

7. L. P. Chew, Constrained Delaunay triangulations, Algorithmica 4 (1989), 97-108.

It is not known whether a constrained Delaunay triangulation for a simple polygon can be computed in less than $O(n \log n)$ time. The following two papers leave open the possibility of the existence of a linear algorithm.

1. A. Aggarwal, L. J. Guibas, J. Saxe, and P. W. Shor, A linear-time algorithm for computing the Voronoi diagram of a convex polygon, Discrete Comput. Geom. 4 (1989), 591-604.

2. B. Chazelle, Triangulating a simple polygon in linear time, Discrete Comput. Geom. 6 (1991), 485-524.

Given a collection of vertices $V$ and edges $E$ such that the endpoints of the edges in $E$ belong to $V$ and two edges in $E$ intersect at most at endpoints, a conforming Delaunay triangulation for $V$ and $E$ is essentially a Delaunay triangulation for a set of points $S$ such that $V$ is contained in $S$ and every edge in $E$ is the union of edges in the triangulation. In the following paper it is proven that given as above sets $V$ and $E$ of $n$ vertices and $m$ edges, respectively, a set $S$ of $O\left(m^{2} n\right)$ points can be obtained in $O\left(m^{2} n+n^{2}\right)$ time such that a 
Delaunay triangulation for $S$ is a conforming Delaunay triangulation for $V$ and $E$, provided infinite precision arithmetic in constant time is assumed.

1. H. Edelsbrunner and T. S. Tan, An upper bound for conforming Delaunay triangulations, Proc. $8^{\text {th }}$ Ann. ACM Symp. on Computational Geometry (1992), 53-62.

\section{Voronoi diagrams for general figures}

The concept of Voronoi diagrams can be generalized to figures more general than points. The following papers present algorithms for exclusively computing the medial axis or Voronoi diagram for the boundary edges of a simple polygon. The second paper presents an $O(n \log n)$ algorithm, where $n$ is the number of boundary edges of the polygon.

1. F. P. Preparata, The medial axis of a simple polygon, Proc. $6^{\text {th }}$ Ann. Internat. Symp. Math. Found. Computer Sci. (1977), 443-450.

2. D. T. Lee, Medial Axis transformation of a planar shape, IEEE Trans. Patt. Anal. Mach. Intell. PAMI-4 (1982), 363-369.

The following paper presents a linear algorithm for computing the medial axis for a convex polygon.

1. A. Aggarwal, L. J. Guibas, J. Saxe, and P. W. Shor, A linear-time algorithm for computing the Voronoi diagram of a convex polygon, Discrete Comput. Geom. 4 (1989), 591-604.

The following paper presents an $O(n \max [\log n, h])$ algorithm for computing the Voronoi diagram for the boundary edges of a multiply connected polygon, where $n$ is the number of boundary edges and $h$ is the number of holes in the polygon.

1. V. Srinivasan and L. R. Nackman, An algorithm to compute the Voronoi diagram of a multiply connected polygonal domain, Research Report, Computer Science, IBM Thomas J. Watson Research Center (1985).

The following papers present $O(n c \sqrt{\log n})$ and $O\left(n \log ^{2} n\right)$ algorithms, respectively, for computing the Voronoi diagram for a set of $n$ disjoint circles and line segments in the plane.

1. R. L. Drysdale and D. T. Lee, Generalized Voronoi diagram in the plane, Proc. $16^{\text {th }}$ Ann. Allerton Conference on Communications, Control and Computing (1978), 833842. 
2. D. T. Lee and R. L. Drysdale, Generalization of Voronoi diagrams in the plane, SIAM J. Comput. 10 (1981), 73-87.

The following paper presents an $O(n \log n)$ algorithm for computing the Voronoi diagram for a set of $n$ line segments in the plane.

1. D. G. Kirkpatrick, Efficient computation of continuous skeletons, Proc. $20^{\text {th }}$ Ann. IEEE Symp. Found. Computer Sci. (1979), 18-27.

The following papers present $O\left(n \log ^{2} n\right)$ divide-and-conquer algorithms for computing, respectively, the Voronoi diagram for a set of $n$ possibly intersecting circles in the plane and the Voronoi diagram for a set of $n$ disjoint planar convex objects of relatively simple shape.

1. M. Sharir, Intersection and closest-pair problems for a set of planar discs, SIAM J. Comput. 14 (1985), 448-468.

2. D. Leven and M. Sharir, Intersection and proximity problems and Voronoi diagrams, Adv. Robotics 1 (1986), 187-228.

In the following paper it is shown that the Voronoi diagram for a set of $n$ spheres in $d$-dimensional space can be obtained linearly from the power diagram for some set of $n$ spheres in $(d+1)$-dimensional space.

1. F. Aurenhammer, Power diagrams: properties, algorithms and applications, SIAM J. Comput. 16 (1987), 78-96.

The following paper presents an $O(n \log n)$ algorithm for computing the Voronoi diagram for a set of $n$ simple curve segments in the plane.

1. C. K. Yap, An $O(n \log n)$ algorithm for the Voronoi diagram of a set of simple curve segments, Discrete Comput. Geom. 2 (1987), 365-393.

The following paper presents $O(n \log n)$ plane-sweep algorithms for computing Voronoi diagrams for sets of $n$ line segments or $n$ possibly intersecting circles in the plane.

1. S. Fortune, A sweepline algorithm for Voronoi diagrams, Algorithmica 2 (1987), 153174.

The following papers address the problem of identifying the Voronoi complex of free placements of a line segment or ladder of length $d$ in the plane for a set of polygonal obstacles. Essentially, this complex consists of all possible line segments of length $d$ in the plane that 
are equidistant from their two closest obtacles. In the first paper the complex is introduced and analyzed. In the second paper it is shown that if the polygonal obstacles have boundaries that consist of $n$ segments and corners then a skeleton representation of the complex can be constructed in time $O\left(n^{2} \log n \alpha(n)^{O\left(\alpha(n)^{\circ}\right)}\right)$ for some fixed integer $s$, where $\alpha(n)$ is the inverse of Ackermann's function.

1. C. Ó'Dúnlaing, M. Sharir, and C. K. Yap, Generalized Voronoi diagrams for moving a ladder. I: Topological analysis, Comm. Pure Appl. Math. 39 (1986), 423-483.

2. C. Ó'Dúnlaing, M. Sharir, and C. Yap, Generalized Voronoi diagrams for a ladder: II. Efficient construction of the diagram, Algorithmica 2 (1987), 27-59.

The first of the following papers presents a randomized incremental construction technique that can be used for computing the Voronoi diagram for a set of $n$ line segments in the plane in $O(n \log n)$ expected time. Based on the same technique, the second paper presents a randomized algorithm for computing in expected $O(n \log n)$ time certain abstract Voronoi diagrams in the plane that include the Voronoi diagram for a set of $n$ line segments and the Voronoi diagram for a set of $n$ disjoint convex objects.

1. K. L. Clarkson and P. W. Shor, Algorithms for diametral pairs and convex hulls that are optimal, randomized, and incremental, Proc. $4^{\text {th }}$ Ann. ACM Symp. on Computational Geometry (1988), 12-17.

2. K. Mehlhorn, C.O'Dunlaing, and S. Meiser, On the construction of abstract Voronoi diagrams, Springer-Verlag Lecture Notes in Computer Science 415 (1990), 227-239.

The following paper presents an algorithm for the parallel computation of the Voronoi diagram for a set of $n$ line segments in the plane in $O\left(\log ^{2} n\right)$ time using $O(n)$ processors.

1. M. T. Goodrich, C. Ó'Dúnlaing, and C. Yap, Constructing the Voronoi diagram of a set of line segments in parallel, Springer-Verlag Lecture Notes in Computer Science 382 (1989), 12-23.

In the following paper the Voronoi diagram for the boundary of a bounded connected set in 3-dimensional space that satisfies a certain set of axioms is considered. The set of axioms has been chosen to produce maximal generality while allowing the definition of Voronoi diagrams that are suitable for motion planning.

1. S. Stifter, An axiomatic approach to Voronoi-diagrams in 3D, J. of Computer and System Sciences 43 (1991), 361-379. 


\section{Voronoi diagrams for general distance measures}

The following papers present $O(n \log n)$ divide-and-conquer algorithms for computing the Voronoi diagram for a set of $n$ points in the plane based on the $L_{1}$ metric, the $L_{1}$ and $L_{\infty}$ metrics, the $L_{p}$ metric, $1 \leq p \leq \infty$, any convex distance function, and any metric induced by a fixed set of orientations, respectively.

1. F. K. Hwang, An $O(n \log n)$ algorithm for rectilinear minimal spanning trees, J. ACM 26 (1979), 177-182.

2. D. T. Lee and C. K. Wong, Voronoi diagrams in $L_{1}\left(L_{\infty}\right)$ metrics with 2-dimensional storage applications, SIAM J. Comput. 9 (1980), 200-211.

3. D. T. Lee, Two-dimensional Voronoi diagrams in the $L_{p}$-metric, J. ACM 27 (1980), 604-618.

4. L. P. Chew and R. L. Drysdale, Voronoi diagrams based on convex distance functions, Proc. $1^{\text {st }}$ Ann. ACM Symp. on Computational Geometry (1985), 235-244.

5. P. Widmayer, Y. F. Wu, and C. K. Wong, On some distance problems in fixed orientations, SIAM J. Comput. 16 (1987), 728-746.

The following paper presents an $O(n \log n)$ divide-and-conquer algorithm for computing a Delaunay triangulation for a set of $n$ points in the plane based on the $L_{p}$ metric, $1<p \leq \infty$, whose expected running time is $O(n \log \log n)$ for any quasi-uniform distribution in a rectangle.

1. R. A. Dwyer, A faster divide-and-conquer algorithm for constructing Delaunay triangulations, Algorithmica 2 (1987), 137-151.

The following paper presents an $O(n \log n)$ plane-sweep algorithm for computing a Delaunay triangulation for a set of $n$ points in the plane based on the $L_{1}$ metric or the $L_{\infty}$ metric.

1. G.M. Shute, L. L. Deneen, and C. D. Thomborson, An $O(n \log n)$ plane-sweep algorithm for $L_{1}$ and $L_{\infty}$ Delaunay triangulations, Tech. Rep. 87-5, Dept. of Comput. Sci., Univ. of Minnesota (1987).

The following paper presents an $O(c n \log c n)$ plane-sweep algorithm for computing the Voronoi diagram for the boundary of a closed set in the plane that has $n$ bounding segments based on a convex distance function defined with respect to a convex polygon that has $c$ bounding edges. 
1. S. J. Fortune, A fast algorithm for polygon containment by translation, Springer-Verlag Lecture Notes in Computer Science 194 (1985), 189-198.

The following paper presents an $O(n \log N)$ algorithm for computing the Voronoi diagram based on a convex distance function for a set of $N$ interior-disjoint convex polygons having $n$ corners.

1. D. Leven and M. Sharir, Planning a purely translational motion for a convex object in two-dimensional space using generalized Voronoi diagrams, Discrete Comput. Geom. 2 (1987), 9-31.

In the following paper it is shown how to compute in an $O(n \log n)$ divide-and-conquer fashion the Voronoi diagram for a set of $n$ points in the plane based on a metric that satisfies a certain set of axioms. The set of axioms has been chosen to ensure that Voronoi diagrams based on such metrics satisfy certain desirable properties. The class of metrics that satisfy the set of axioms contains all symmetric convex distance functions and many combinations of well-known metrics.

1. R. Klein and D. Wood, Voronoi diagrams based on general metrics in the plane, Springer-Verlag Lecture Notes in Computer Science 294 (1988), 281-291.

In the following textbook it is shown how to compute in an $O(n \log n)$ divide-and-conquer fashion, relative to the complexity of elementary bisector operations, the Voronoi diagram for a set of $n$ sites based on a 'nice' metric that admits acyclic partitions of the set of sites. One such metric is the Moscow metric which is defined by the regular part of the fan-shaped street layout of Moscow and for which elementary bisector operations can be carried out in constant time.

1. R. Klein, Concrete and abstract Voronoi diagrams, Springer-Verlag Lecture Notes in Computer Science 400 (1989).

The following paper presents a randomized algorithm for computing in expected $O(n \log n)$ time certain abstract Voronoi diagrams in the plane that include the Voronoi diagram for a set of $n$ points in the plane based on the $L_{p}$ metric with additive weights, $1 \leq p \leq \infty$, any convex distance function, and the Moscow metric, and the Voronoi diagram based on a convex distance function for a set of disjoint convex polygons having $n$ corners.

1. K. Mehlhorn, C.O'Dunlaing, and S. Meiser, On the construction of abstract Voronoi diagrams, Springer-Verlag Lecture Notes in Computer Science 415 (1990), 227-239. 
In the following paper Voronoi or Dirichlet tessellations of simply connected complete riemannian manifolds without conjugate points are investigated.

1. P.E. Ehrlich and H.-C. Im Hof, Dirichlet regions in manifolds without conjugate points, Comment. Math. Helvetici 54 (1979), 642-658.

In the following paper expectations of random variables concerning the Voronoi diagram for a finite random set of points on the surface of a 3-dimensional sphere are derived. Here the length of the shortest geodesic between two points is used as the measure of distance.

1. R. E. Miles, Random points, sets and tessellations on the surface of a sphere, Sankhyā: The Indian Journal of Statistics: Series A 33 (1971), 145-174.

The following papers present incremental diagonal-flipping algorithms for computing a Delaunay triangulation for a set of points on the surface of a 3-dimensional sphere. Here the edges of the triangles are geodesics and an empty circle criterion in the spherical setting is used for choosing, whenever necessary, between the two possible dissections of a strictly convex spherical quadrilateral.

1. C. L. Lawson, $C^{1}$ surface interpolation for scattered data on a sphere, Rocky Mountain J. of Mathematics 14 (1984), 177-202.

2. R. J. Renka, Interpolation of data on the surface of a sphere, ACM Transactions on Mathematical Software 10 (1984), 417-436, 437-439.

The following paper presents an algorithm for computing the Voronoi diagram for a set of sites lying on the surface of a possibly nonconvex 3-dimensional polyhedron, using the Euclidean length of the shortest path between two points along the surface of the polyhedron as the measure of distance. The running time of the algorithm is $O\left(n^{2} \log n\right)$, where $n$ is the maximum of the number of edges and sites on the polyhedron. The output of the program requires $O\left(n^{2}\right)$ space.

1. D. M. Mount, Voronoi diagrams on the surface of a polyhedron, CS-TR-1496, Dept. of Comput. Sci., Univ. of Maryland (1985).

The following paper presents an $O(n \log n)$ sweepcircle algorithm for computing the Voronoi diagram for a set of $n$ sites lying on the surface of a 3 -dimensional cone, using the minimum Euclidean length of all curves on the surface of the cone that connect two points as the measure of distance. The algorithm uses a circle that emanates from the peak of the cone for computing a certain transformation of the Voronoi diagram that preserves its combinatorial structure. 
1. F. Dehne and R. Klein, An optimal algorithm for computing the Voronoi diagram on a cone, SCS-TR-122, School of Comput. Sci., Carleton Univ. (1987).

The third of the following papers shows how to compute in $O(m n+m \log \log m+n \log n)$ time and $O(m+n)$ space the geodesic Voronoi diagram for a set of $n$ points in a simple polygon of $m$ vertices in the plane, using the length of the shortest internal path between two points in the polygon as the measure of distance. The method is based on an algorithm in the second paper that can be used to compute in $O(m n+m \log \log m)$ time and $O(m+n)$ space the shortest path trees from the $n$ points to the vertices of the polygon. It is also observed in the third paper that in the presence of polygonal obstacles of $m$ vertices, the geodesic Voronoi diagram for a set of $n$ points in the plane can be computed in a similar manner in $O\left(m^{2}+m n+n \log n\right)$ time and $O\left(m^{2}+n\right)$ space. The method uses an algorithm in the first paper for finding a Euclidean shortest path between two points in the presence of polygonal obstacles.

1. T. Asano, T. Asano, L. Guibas, J. Hershberger, and H. Imai, Visibility of disjoint polygons, Algorithmica 1 (1986), 49-63.

2. L. Guibas, J. Hershberger, D. Leven, M. Sharir, and R. E. Tarjan, Linear-time algorithms for visibility and shortest path problems inside a triangulated simple polygon, Algorithmica 2 (1987), 209-233.

3. T. Asano and T. Asano, Voronoi diagram for points in a simple polygon, Perspectives in Computing, Discrete Algorithms and Complexity, Proc. Japan-US Joint Seminar, 1986, D. S. Johnson, T. Nishizeki, A. Nozaki, H. S. Wilf (Eds.), Academic Press, New York (1987), 51-64.

Employing a divide-and-conquer strategy, the following paper presents an algorithm for computing in $O((n+k) \log (n+k) \log n)$ time the geodesic Voronoi diagram for a set of $k$ points in a simple polygon with $n$ sides in the plane, using the length of the shortest internal path between two points in the polygon as the measure of distance. It is also shown in this paper that the worst-case total size of this diagram is $O(n+k)$, and a simplified version of the algorithm is sketched that computes in $O((n+k) \log (n+k))$ time the diagram for a set of points that contains all reflex vertices of the polygon. In particular it is observed in this paper that the simpler algorithm can be used to compute the geodesic Voronoi diagram for the vertices of a simple polygon with $n$ vertices in $O(n \log n)$ time, and that the dual of this diagram is essentially the constrained Delaunay triangulation for the polygon.

1. B. Aronov, On the geodesic Voronoi diagram of point sites in a simple polygon, Algorithmica 4 (1989), 109-140. 
The following paper addresses the problem of identifying the Voronoi complex of free placements of an arbitrary 3-dimensional polyhedron $A$ for a set of 3-dimensional polyhedral obstacles based on a particular measure of distance $\rho$ which is not a true metric. Essentially, this complex consists of all possible 3 -dimensional polyhedra that can be obtained by translating and/or rotating $A$ and that are equidistant under $\rho$ from their two closest obstacles.

1. J. Canny and B. Donald, Simplified Voronoi diagrams, Discrete Comput. Geom. 3 (1988), 219-236.

The following paper presents an $O(n \log n)$ divide-and-conquer algorithm for computing a convex shape Delaunay triangulation for a set of $n$ sites in the plane. Given a convex shape, a convex shape Delaunay triangulation for a set of sites in the plane is a maximal graph in which each node is a site and each edge intersects no other edges or sites except at nodes, and for which, given an edge, it is possible to scale and/or translate the convex shape so that both nodes of the edge are on the boundary of the shape while no other site is in its interior.

1. R. L. Drysdale, A practical algorithm for computing the Delaunay triangulation for convex distance functions, Proc. $1^{\text {st }}$ Ann. ACM-SIAM Symp. on Discrete Algorithms (1990), 159-168.

\section{Furthest point Voronoi diagrams}

The following papers present methods for computing in $O(n \log n)$ time furthest point Voronoi diagrams for sets of $n$ points in the plane, i. e. order $-(n-1)$ Voronoi diagrams.

1. M. I. Shamos and D. Hoey, Closest-point problems, Proc. $16^{\text {th }}$ Ann. IEEE Symp. on Foundations of Computer Science (1975), 151-162.

2. F. P. Preparata, Steps into computational geometry, Tech. Rep., Coordinated Science Laboratory, University of Illinois (1977).

3. M. I. Shamos, Computational geometry, Ph. D. thesis, Dept. of Comput. Sci., Yale Univ. (1978).

4. D. T. Lee, Farthest neighbor Voronoi diagrams and applications, Tech. Rep. 80-11FC-04, Dept. of Elec. Eng. and Comput. Sci., Northwestern Univ. (1980). 
In the following paper geometrical properties of the furthest point Voronoi diagram in the plane are derived.

1. B. K. Bhattacharya and G. T. Toussaint, On geometric algorithms that use the furthest point Voronoi diagram, Computational Geometry, G. T. Toussaint (Ed.), NorthHolland (1985), 43-61.

In the following paper it is shown how to maintain dynamically a furthest point Voronoi diagram for $n$ points in $O(n)$ time per insertion and $O(n \log n / k(n))$ time per deletion using $O(n \log (k(n)))$ storage, where $k(n)=O(\log n)$.

1. I. G. Gowda, D. G. Kirkpatrick, D. T. Lee, and A. Naamad, Dynamic Voronoi diagrams, IEEE Transactions on Information Theory IT-29 (1983), 724-731.

In the first of the following papers an asymptotically tight upper bound is derived for the number of $i$-dimensional faces of furthest point Voronoi diagrams for $n$ points in $d$-dimensional space, for each $i, i=0, \ldots, d-1$. In the second paper exact upper bounds are derived.

1. R. Seidel, The complexity of Voronoi diagrams in higher dimensions, Proc. $20^{\text {th }}$ Ann. Allerton Conference on Communication, Control, and Computing (1982), 94-95.

2. R. Seidel, On the number of faces in higher-dimensional Voronoi diagrams, Proc. $3^{\text {rd }}$ Ann. ACM Symp. on Computational Geometry (1987), 181-185.

In the third of the following papers it is shown how to compute in $O\left(n^{3} \log \log n\right)$ time the furthest point geodesic Voronoi diagram for the vertices of a simple polygon, where $n$ is the number of vertices, using the length of the shortest internal path between two points in the polygon as the measure of distance. The method is based on an $O(n \log \log n)$ algorithm in the second paper for determining which points in the polygon are further from one of two given points in the polygon, which itself is based on an $O(n \log \log n)$ algorithm in the first paper for computing a shortest path tree from a point in the polygon to the vertices of the polygon.

1. L. Guibas, J. Hershberger, D. Leven, M. Sharir, and R. E. Tarjan, Linear-time algorithms for visibility and shortest path problems inside a triangulated simple polygon, Algorithmica 2 (1987), 209-233.

2. T. Asano and T. Asano, Voronoi diagram for points in a simple polygon, Perspectives in Computing, Discrete Algorithms and Complexity, Proc. Japan-US Joint Seminar, 1986, D. S. Johnson, T. Nishizeki, A. Nozaki, H. S. Wilf (Eds.), Academic Press, New York (1987), 51-64. 
3. T. Asano and G. Toussaint, Computing the geodesic center of a simple polygon, Perspectives in Computing, Discrete Algorithms and Complexity, Proc. Japan-US Joint Seminar, 1986, D. S. Johnson, T. Nishizeki, A. Nozaki, H. S. Wilf (Eds.), Academic Press, New York (1987), 65-79.

The following paper presents an algorithm for computing in $O((n+k) \log (n+k))$ time and $O(n+k)$ space the furthest point geodesic Voronoi diagram for a set of $k$ points in a simple polygon with $n$ sides in the plane, using the length of the shortest internal path between two points in the polygon as the measure of distance.

1. B. Aronov, S. Fortune, and G. Wilfong, The furthest-site geodesic Voronoi diagram, Proc. $4^{\text {th }}$ Ann. ACM Symp. on Computational Geometry (1988), 229-240.

In the following paper a linear time algorithm for computing the furthest point Voronoi diagram for the vertices of a convex polygon is discussed.

1. A. Aggarwal, L. J. Guibas, J. Saxe, and P. W. Shor, A linear-time algorithm for computing the Voronoi diagram of a convex polygon, Discrete Comput. Geom. 4 (1989), 591-604.

The following paper presents a general method for determining the expected complexity of further point Voronoi diagrams for random point sets in $d$-dimensional space.

1. R. A. Dwyer, Higher-dimensional Voronoi diagrams in linear expected time, Discrete Comput. Geom. 6 (1991), 343-367.

In the following paper it is proven that among all triangulations for a set of points in general convex position, the furthest point Delaunay triangulation has the lexicographically smallest increasing sequence of interior angles of triangles. A set of points is in general convex position if the points form the vertices of a convex polygon and no four of them are cocircular.

1. D. Eppstein, The farthest point Delaunay triangulation minimizes angles, Computational Geometry: Theory and Applications 1 (1992), 143-148.

The following paper presents an algorithm for computing in $O(n \log n)$ time the furthest point Voronoi diagram for a set of $n$ discs in the plane.

1. D. Rappaport, A convex hull algorithm for discs, and applications, Computational Geometry: Theory and Applications 1 (1992), 171-187. 


\section{Order $-k$ Voronoi diagrams}

In the following papers expectations of random variables concerning order $-k$ Voronoi diagrams for stationary Poisson point processes in 2- and 3-dimensional spaces are discussed.

1. R. E. Miles, On the homogeneous planar Poisson point process, Mathematical Biosciences 6 (1970), 85-127.

2. R. E. Miles, The random division of space, Proc. Symp. on Statistical and Probabilistic Problems in Metallurgy, August 1971, Suppl. Adv. Appl. Prob. (1972), 243-266.

In the following paper the total number of bounded and unbounded Voronoi regions in all of the $k$-order Voronoi diagrams, $k=1, \ldots, n-1$, for a set of $n$ points in the plane is computed.

1. M. I. Shamos and D. Hoey, Closest-point problems, Proc. $16^{\text {th }}$ Ann. IEEE Symp. on Foundations of Computer Science (1975), 151-162.

The first of the following papers presents an algorithm for computing the order $-k$ Voronoi diagram for a set of $n$ points in the plane, $1 \leq k \leq n-1$, in $O\left(k^{2} n \log n\right)$ time and $O\left(k^{2}(n-k)\right)$ space. Using a linear procedure presented in the second paper for eliminating a site from a planar order-1 Voronoi diagram, the time of this algorithm can be reduced to $O\left(n k^{2}+n \log n\right)$. The algorithm computes the order $-k$ Voronoi diagram from the order $-(k-1)$ Voronoi diagram for the set. Exact upper bounds for the complexity of order $-k$ Voronoi diagrams in the plane are also established in the first paper.

1. D. T. Lee, On $k$-nearest neighbor Voronoi diagrams in the plane, IEEE Trans. Comput. C-31 (1982), 478-487.

2. A. Aggarwal, L. J. Guibas, J. Saxe, and P. W. Shor, A linear-time algorithm for computing the Voronoi diagram of a convex polygon, Discrete Comput. Geom. 4 (1989), 591-604.

The following paper presents an algorithm for computing in $O\left(n^{4}\right)$ time all order- $k$ Voronoi diagrams for a set of $n$ points in the plane, $k=1, \ldots, n-1$. The algorithm is optimal if explicit lists of closest points are required for each region in each order $-k$ Voronoi diagram, $k=1, \ldots, n-1$.

1. F. Dehne, An optimal algorithm to construct all Voronoi diagrams for $k$ nearest neighbor searching in the Euclidean plane, Proc. $20^{\text {th }}$ Ann. Allerton Conference on Communication, Control, and Computing (1982), 85-93. 
The following paper presents an $O\left(k n^{2}\right)$ algorithm for computing directly the order $-k$ Voronoi diagram for a set of $n$ points in the plane and an arbitrary value of $k, 1 \leq k \leq n-1$. The space requirement is $O\left(k^{2} n\right)$ if explicit lists of closest points are required for each region in the order $-k$ Voronoi diagram, $O(k n)$ otherwise.

1. B. Bhattacharya, An algorithm for computing order $k$ Voronoi diagrams in the plane, Tech. Rep. 83-9, Comput. Sci. Dept., Simon Fraser Univ. (1983).

The following paper presents an optimal algorithm for computing in $O\left(n^{d}\right)$ time the arrangement for $n$ hyperplanes in $d$-dimensional space, $d \geq 2$. It implies an optimal algorithm for computing in $O\left(n^{d+1}\right)$ time all order $-k$ Voronoi diagrams for a set of $n$ points in $d$-dimensional space, $d \geq 2, k=1, \ldots, n-1$. The algorithm requires a geometric transformation that maps the set of points to a set of hyperplanes in $(d+1)$-dimensional space. All order $-k$ Voronoi diagrams are then obtained by computing the arrangement of the set of hyperplanes and then projecting vertically all levels of all orders in the arrangement onto $d$-dimensional space. A level of order $k$ in an arrangement is essentially the collection of cells in the arrangement that lie below $k$ and above $n-k$ hyperplanes.

1. H. Edelsbrunner, J. O'Rourke, and R. Seidel, Constructing arrangements of lines and hyperplanes with applications, SIAM J. Comput. 15 (1986), 341-363.

The following papers present algorithms for computing the order- $k$ Voronoi diagram for a set of $n$ points in the plane, $1 \leq k \leq n-1$. All algorithms require a geometric transformation that maps the set of points to a set of planes in 3 -dimensional space. The order- $k$ Voronoi diagram is then obtained by computing certain edges in the arrangement of these planes and then projecting them vertically onto the plane. The first paper presents an algorithm that requires $O(k(n-k) \sqrt{n} \log n)$ time and $O(k(n-k))$ space. The second paper presents two versions of an algorithm that require $O\left(n^{2} \log n+k(n-k) \log ^{2} n\right)$ time and $O(k(n-k))$ space, and $O\left(n^{2}+k(n-k) \log ^{2} n\right)$ time and $O\left(n^{2}\right)$ space, respectively. This paper also discusses a generalization of this algorithm for computing the order $-k$ Voronoi diagram for a set of $n$ points in 3 -dimensional space, $1 \leq k \leq n-1$, in $O\left(n^{2} \log n+c(n) \sqrt{n} \log n\right)$ time and $O(c(n))$ space, where $c(n)$ is the maximum number of edges in an order $-k$ Voronoi diagram for a set on $n$ points.

1. H. Edelsbrunner, Edge-skeletons in arrangements with applications, Algorithmica 1 (1986), 93-109.

2. B. Chazelle and H. Edelsbrunner, An improved algorithm for constructing $k^{\text {th }}$-order Voronoi diagrams, IEEE Trans. Comput. C-36 (1987), 1349-1354. 
The following paper presents an algorithm for computing the order- $k$ Voronoi diagram for a set of $n$ points in $d$-dimensional space, $1 \leq k \leq n-1$, which is space optimal for any $d$ and that requires $O\left(k^{2} n \log n\right)$ time for $d$ equal to 2. For each $k$ the algorithm associates the set of $n$ points to a dual set $Q_{k}$ in $(d+1)$-dimensional space. The order- $k$ Voronoi diagram is then obtained by computing the lower part of the convex hull of $Q_{k}$ and then dualizing it to $d$-dimensional space. The algorithm computes the lower part of the convex hull of $Q_{k}$ from the lower part of the convex hull of $Q_{k-1}$.

1. F. Aurenhammer, A new duality result concerning Voronoi diagrams, Discrete Comput. Geom. 5 (1990), 243-254.

In the following paper, given $\epsilon>0$, an algorithm is discussed for computing the order $-k$ Voronoi diagram for a set $S$ of $n$ points in the plane, $1 \leq k \leq n-1$, in expected $O\left(n^{1+\epsilon} k\right)$ time. It is assumed that no four points in $S$ are cocircular. The algorithm associates the set $S$ to a set $S^{\prime}$ in 3-dimensional space and through the use of random sampling computes certain triples of points in $S^{\prime}$ that are closely related to $k$-sets of $S^{\prime}$ from which the order- $k$ Voronoi diagram can be computed. A $k$-set of $S^{\prime}$ is a subset of $S^{\prime}$ of size $k$ that is all on one side of some hyperplane, while the other points in $S^{\prime}$ are all on the other side of the hyperplane.

1. K. L. Clarkson, New applications of random sampling in computational geometry, Discrete Comput. Geom. 2 (1987), 195-222.

The following paper presents a randomized algorithm for computing all Voronoi diagrams of order 1 to $k, 1 \leq k \leq n-1$, in $d$-dimensional space in expected time $O\left(k^{\lceil(d+1) / 2\rceil} n^{\lfloor(d+1) / 2\rfloor}\right)$ for $d \geq 3$ and $O\left(n k^{2}+n \log n\right)$ for $d=2$. The algorithm computes these diagrams by essentially computing levels of order 1 to $k$ in some specialized arrangement in $(d+1)$-dimensional space.

1. K. Mulmuley, On levels in arrangements and Voronoi diagrams, Discrete Comput. Geom. 6 (1991), 307-338.

The following paper presents an on-line randomized incremental algorithm for computing the order $-k$ Voronoi diagram for a set of $n$ points in the plane, $1 \leq k \leq n-1$, in expected time $O\left(k^{2}(n-k) \log n+n k \log ^{3} n\right)$ and optimal worst-case space $O(k(n-k))$. It is also shown in this paper how this algorithm can be speeded up to have an optimal expected time complexity of $O\left(k^{2}(n-k)+n k \log ^{2} n\right)$ without affecting the space complexity. The algorithm requires a duality transform that allows the insertion and deletion of sites in the diagram in a simple fashion through the computation of 3-dimensional convex hulls. 
1. F. Aurenhammer and O. Schwarzkopf, A simple on-line randomized incremental algorithm for computing higher order Voronoi diagrams, Proc. $7^{\text {th }}$ Ann. ACM Symp. on Computational Geometry (1991), 142-151.

\section{Multiplicative and additively weighted Voronoi diagrams}

In the following papers expectations of random variables concerning additively weighted Voronoi diagrams for stationary Poisson point processes in 2- and 3-dimensional spaces are derived.

1. J. L. Meijering, Interface area, edge length, and number of vertices in crystal aggregates with random nucleation, Philips Res. Rep. 8 (1953), 270-290.

2. E. N. Gilbert, Random subdivisions of space into crystals, Ann. Math. Statist. 33 (1962), 958-972.

3. R. E. Miles, The random division of space, Proc. Symp. on Statistical and Probabilistic Problems in Metallurgy, August 1971, Suppl. Adv. Appl. Prob. (1972), 243-266.

The first of the following papers presents an optimal $O\left(n^{2}\right)$ algorithm for computing the multiplicative weighted Voronoi diagram for $n$ points with positive weights in the plane. The second paper presents an optimal $O(n \log n)$ algorithm for computing the multiplicative weighted Voronoi diagram for $n$ points with positive weights on a line.

1. F. Aurenhammer and H. Edelsbrunner, An optimal algorithm for constructing the weighted Voronoi diagram in the plane, Pattern Recognition 17 (1984), 251-257.

2. F. Aurenhammer, The one-dimensional weighted Voronoi diagram, Inf. Proc. Letters 22 (1986), 119-123.

In the following paper a criterion is given for recognizing certain multiplicative and additively weighted Voronoi diagrams in the plane, and for computing their sources and weights.

1. P. F. Ash and E. D. Bolker, Generalized Dirichlet tessellations, Geometriae Dedicata 20 (1986), 209-243.

In the first of the following papers it is shown that the (order-1) additively weighted Voronoi diagram for a set of $d$-dimensional $n$ points with weights can be obtained linearly from the (order-1) power diagram for some set of $n$ spheres in $(d+1)$-dimensional space. In this paper it is also shown that all order $-k$ multiplicative weighted Voronoi diagrams for 
a set of $d$-dimensional $n$ points with positive weights, $k=1, \ldots, n-1$, can be obtained linearly from the order $-k$ power diagrams for some set of $n$ spheres in $(d+1)$-dimensional space, $k=1, \ldots, n-1$. In the second paper a similar result is presented for computing multiplicative as well as additively weighted Voronoi diagrams of all orders in $d$-dimensional space, and in addition it is shown how to compute efficiently in $d$-dimensional space an order $-k$ power diagram as an (order -1 ) power diagram.

1. F. Aurenhammer, Power diagrams: properties, algorithms and applications, SIAM J. Comput. 16 (1987), 78-96.

2. F. Aurenhammer and H. Imai, Geometric relations among Voronoi diagrams, Geometriae Dedicata 27 (1988), 65-75.

The following papers present, respectively, an $O\left(n \log ^{2} n\right)$ divide-and-conquer algorithm and an $O(n \log n)$ plane-sweep algorithm for computing the additively weighted or hyperbolic Voronoi diagram for $n$ points with weights in the plane or, equivalently, the Voronoi diagram for $n$ possibly intersecting circles in the plane.

1. M. Sharir, Intersection and closest-pair problems for a set of planar discs, SIAM J. Comput. 14 (1985), 448-468.

2. S. Fortune, A sweepline algorithm for Voronoi diagrams, Algorithmica 2 (1987), 153174 .

The following paper presents a randomized algorithm for computing in expected $O(n \log n)$ time certain abstract Voronoi diagrams in the plane that include the Voronoi diagram for a set of $n$ points in the plane based on the $L_{p}$ metric with additive weights, $1 \leq p \leq \infty$.

1. K. Mehlhorn, C.O'Dunlaing, and S. Meiser, On the construction of abstract Voronoi diagrams, Springer-Verlag Lecture Notes in Computer Science 415 (1990), 227-239.

The following paper presents a plane-sweep type algorithm for computing the order $-k$ Voronoi diagram for a set of $n$ points with additive weights in the plane, $1 \leq k \leq n-1$, in $O\left(k^{2} n \log n\right)$ time and $O(k n)$ space. The algorithm is based on a relationship between order $-k$ Voronoi diagrams for points with additive weights in the plane and certain arrangements of cones in 3-dimensional space. Upper bounds for the complexity of order $-k$ Voronoi diagrams with additive weights in the plane are also established in this paper.

1. H. Rosenberger, Order $-k$ Voronoi diagrams of sites with additive weights in the plane, Algorithmica 6 (1991), 490-521. 


\section{Power diagrams and arrangements}

The following paper presents an $O(n \log n)$ algorithm for computing the power diagram for $n$ circles in the plane. It is also shown in this paper that a planar power diagram is the intersection of the $x-y$ plane and the Voronoi diagram for a set of points in 3-dimensional space whose perpendicular projections on the $x-y$ plane are the centers of the circles, and vice versa.

1. H. Imai, M. Iri, and K. Murota, Voronoi diagram in the Laguerre geometry and its applications, SIAM J. Comput. 14 (1985), 93-105.

The following paper presents an incremental triangle-flipping algorithm for computing a regular triangulation, i. e. the dual of a power diagram, for a set of $n$ points in $d$-space in expected $O\left(n \log n+n^{[d / 2\rceil}\right)$ time if the points are added in a random sequence.

1. H. Edelsbrunner and N. R. Shah, Incremental topological flipping works for regular triangulations, Proc. $8^{\text {th }}$ Ann. ACM Symp. on Computational Geometry (1992), 43-52.

In the following paper Voronoi diagrams in the plane are generalized through the distortion of the Euclidean distance by a smooth increasing function and the weighting of sites by additive constants. It is shown in this paper that 2-dimensional ordinary and hyperbolic Voronoi diagrams or Dirichlet tessellations, power diagrams or sectional Dirichlet tessellations, and multiplicative weighted Voronoi diagrams or circular Dirichlet tessellations satisfy this definition for the proper choice of the distorting function and weighting constants. Criteria are given in this paper for recognizing some of these generalized Dirichlet tessellations and a study is presented of how the choice of the distorting function affects the shapes of the regions in the tessellation.

1. P. F. Ash and E. D. Bolker, Generalized Dirichlet tessellations, Geometriae Dedicata 20 (1986), 209-243.

In the following paper a general definition for ordinary and higher order Voronoi diagrams in $d$-dimensional space is presented in terms of arrangements in $(d+1)$-dimensional space, i. e. partitions of $(d+1)$-dimensional space induced by a finite collection of real-valued functions on $d$-dimensional space. In this definition no notion of a metric is required. It is shown in this paper that ordinary, order $-k$, degree- $k$ Voronoi diagrams, and power diagrams in $d$-dimensional space satisfy this definition in terms of arrangements of hyperplanes. Consequently, algorithms for computing intersections of half-spaces and arrangements of hyperplanes can be used for computing all of these diagrams. 
1. H. Edelsbrunner and R. Seidel, Voronoi diagrams and arrangements, Discrete Comput. Geom. 1 (1986), 25-44.

The following paper presents a plane-sweep algorithm for computing the arrangement for a set of hyperplanes in $d$-dimensional space.

1. H. Bieri and W. Nef, A recursive sweep-plane algorithm, determining all cells of a finite division of $R^{d}$, Computing 28 (1982), 189-198.

The following paper presents an $O\left(n^{2} \log n\right)$ plane-sweep algorithm for computing the arrangement for a set of $n$ lines in the plane.

1. H. Edelsbrunner and E. Welzl, Constructing belts in two-dimensional arrangements with applications, SIAM J. Comput. 15 (1986), 271-284.

The following paper presents an optimal algorithm for computing in $O\left(n^{2}\right)$ time the arrangement for $n$ lines in the plane.

1. B. Chazelle, L. J. Guibas, and D. T. Lee, The power of geometric duality, BIT 25 (1985), 76-90.

The following paper presents an optimal algorithm for computing in $O\left(n^{d}\right)$ time the arrangement for $n$ hyperplanes in $d$-dimensional space, $d \geq 2$.

1. H. Edelsbrunner, J. O'Rourke, and R. Seidel, Constructing arrangements of lines and hyperplanes with applications, SIAM J. Comput. 15 (1986), 341-363.

In the following papers it is shown that a power diagram for a set of $d$-dimensional spheres is obtained by projecting vertically onto $d$-space the boundary of a $(d+1)$-dimensional polyhedron which is expressible as the intersection of upper halfspaces. It is also shown in these papers that every power diagram can be obtained in this fashion. In the second paper power diagrams are generalized to higher order and it is shown that $d$-dimensional maximal power diagrams, i. e. order- $(n-1)$ power diagrams for sets of $n d$-dimensional spheres, can also be characterized in terms of convex polyhedral surfaces in $(d+1)$-dimensional spaces. In addition, it is shown in this paper that the power diagram and the maximal power diagram for a set of spheres in $d$-dimensional space are dual to the lower and upper parts, respectively, of the convex hull of some finite set of points in $(d+1)$-dimensional space, and vice versa. Moreover, it is shown in this paper that the collection of power diagrams of all orders for a set of $n d$-dimensional spheres corresponds to a $(d+1)$-dimensional arrangement of hyperplanes and vice versa, and that in particular the order $-k$ power diagram for the set 
of spheres is the vertical projection of the intersection of levels of order $k$ and $k+1$ in the arrangement, for each $k, k=1, \ldots, n-1$. From these results it follows that the order -1 and the maximal power diagrams for a set of $d$-dimensional spheres can be obtained through the computation of either a convex hull or certain intersections of hyperplanes in $(d+1)-$ dimensional space, and that all higher order power diagrams for the set of spheres can be obtained through the computation of a $(d+1)$-dimensional arrangement of hyperplanes. Finally, it is shown in this paper that given a set $S$ of $n$ spheres in $d$-dimensional space there is a set $S^{*}$ of $n$ spheres in $d$-dimensional space such that the order-k power diagram for $S$ equals the order- $(n-k)$ power diagram for $S^{*}$ for each $k, k=1, \ldots, n-1$. From this result it follows in particular that ordinary power diagrams and maximal power diagrams have the same complexity.

1. I. Paschinger, Konvexe Polytope und Dirichletsche Zellenkomplexe, Ph. D. dissertation, Math. Inst., Univ. Salzburg, Austria (1982).

2. F. Aurenhammer, Power diagrams: properties, algorithms and applications, SIAM J. Comput. 16 (1987), 78-96.

In the first and second of the following papers it is shown that a cell complex in 2-dimensional space is the power diagram for some set of circles if and only if it has an orthogonal dual. In the second paper this result is generalized to $d$-dimensional space, $d \geq 1$. Using this result it is shown in this paper that order $-k$ power diagrams and arrangements are (order -1 ) power diagrams for $d \geq 1$, and a new proof is presented for the fact that simple cell complexes are (order-1) power diagrams for $d \geq 3$. A cell complex in $d$-dimensional space is simple if each of its vertices is a vertex of exactly $d+1$ cells in the cell complex. Under the requirement that a cell complex be given by its incidence matrix, the third paper presents an algorithm for deciding whether a simple $d$-dimensional cell complex is an (order-1) power diagram for $d=2$, and for computing a $(d+1)$-dimensional polyhedron if it exists whose perpendicular projection is the cell complex for $d \geq 2$. Essentially, the algorithm computes an orthogonal dual for the cell complex if it exists in time proportional to the number of facets of the cell complex, and from this dual computes the $(d+1)$-dimensional polyhedron.

1. P. F. Ash and E. D. Bolker, Generalized Dirichlet tessellations, Geometriae Dedicata 20 (1986), 209-243.

2. F. Aurenhammer, A criterion for the affine equivalence of cell complexes in $R^{d}$ and convex polyhedra in $R^{d+1}$, Discrete Comput. Geometry 2 (1987), 49-64.

3. F. Aurenhammer, Recognising polytopical cell complexes and constructing projection polyhedra, J. Symbolic Comput. 3 (1987), 249-255. 
In the following paper affine Voronoi diagrams for sets of sites in $d$-dimensional space, i. e. Voronoi diagrams based on distance functions for which each set of points in the space equidistant from two sites forms a hyperplane, are shown to be power diagrams. In addition, affinely transformable Voronoi diagrams for sets of sites in $d$-dimensional space, i. e. Voronoi diagrams based on distance functions for which each set of points in the space equidistant from two sites can be transformed in a certain way into a set in $(d+1)$-dimensional space whose affine hull is a hyperplane, are shown to be perpendicular projections of certain sections of power diagrams in $(d+1)$-dimensional space. In particular, it is shown that multiplicative and additively weighted Voronoi diagrams for sets of $n$ points in $d$-dimensional space are affinely transformable and can be obtained linearly from power diagrams for the corresponding sets of $n$ points and power functions in $(d+1)$-dimensional space. It is also shown in this paper that given a $d$-dimensional set $G$ of $n$ sites and a power function $f$ for $G$, for each $k, k=2, \ldots, n-1$, by letting $\Gamma_{1}=G$ and $\phi_{1}=f$, a set of generators $\Gamma_{k}$ and a power function $\phi_{k}$ for $\Gamma_{k}$ can be computed iteratively and linearly from the power diagram for $\Gamma_{k-1}$ based on $\phi_{k-1}$, in such a way that the order $-k$ power diagram for $G$ based on $f$ equals the (order-1) power diagram for $\Gamma_{k}$ based on $\phi_{k}$, and the size of $\Gamma_{k}$ does not exceed the maximal number of $d$-dimensional cells that a $d$-dimensional order $-k$ power diagram for $n$ points can have. Finally, it is shown in this paper that order- $k$ Voronoi diagrams for sets in $d$-dimensional space are perpendicular projections of certain sections of order- $k$ power diagrams in $(d+1)$-dimensional space if they are based on distance functions for which the (order-1) Voronoi diagram is affinely transformable. In particular, this result implies methods for computing linearly order $-k$ multiplicative and additively weighted Voronoi diagrams for sets of $n$ points in $d$-dimensional space from order- $k$ power diagrams for the corresponding sets of $n$ points and power functions in $(d+1)$-dimensional space.

1. F. Aurenhammer and H. Imai, Geometric relations among Voronoi diagrams, Geometriae Dedicata 27 (1988), 65-75.

In the following paper the relationship between the power diagram for a set of discs or balls in $d$-dimensional space and computations that concern their union and intersection is demonstrated.

1. F. Aurenhammer, Improved algorithms for discs and balls using power diagrams, J. of Algorithms 9 (1988), 151-161.

In the following paper it is shown that given a cell complex in $d$-dimensional space that is the vertical projection onto $d$-dimensional space of the lower boundary of a $(d+1)$-dimensional polytope, for each point in the space there exists a partial ordering of the cells that is consistent with the order in which the cells are visible from the point. 
1. H. Edelsbrunner, An acyclicity theorem for cell complexes in $d$ dimension, Proc. $5^{\text {th }}$ Ann. ACM Symp. on Computational Geometry (1989), 145-151.

In the first of the following papers it is shown that given a set of points $S$ in $d$-dimensional space with nonnegative weights, every point $p$ in $S$ whose power cell in the power diagram for $S$ is bounded and nonempty can be expressed as a convex combination of the other points in $S$, in such a way that given $q$ in $S, q \neq p$, the coefficient that corresponds to $q$ in this convex combination is the percentage of the power cell of $p$ that is more under the power of $q$ than under the power of any other point in $S \backslash\{p, q\}$. In the second paper this result is generalized to order $-k$ power cells, $1 \leq k \leq n-1$. In the third paper it is shown how these combinations can be used to compute a Gale transform for $S$ if the (order-1) power cell for each point in $S$ in the (order-1) power diagram for $S$ is nonempty.

1. R. Sibson, A vector identity for the Dirichlet tessellation, Math. Proc. Camb. Phil. Soc. 87 (1980), 151-155.

2. F. Aurenhammer, Linear combinations from power domains, Geometriae Dedicata 28 (1988), 45-52.

3. F. Aurenhammer, A relationship between Gale transforms and Voronoi diagrams, Discrete Applied Mathematics 28 (1990), 83-91.

In the following paper it is shown that an assignment induced by a power diagram in $d$-dimensional space is a least-squares assignment, i. e. given a set $S$ of sites and a finite or bounded measurable set of points $X$ in $d$-dimensional space, among all functions that assign a site in $S$ to each point in $X$ and that according to the site assignment partition $X$ into subsets of prescribed sizes (capacities), for some set of weights $W$, the function induced in the obvious manner by the power diagram for $S$ with weights $W$ minimizes the sum or integral of the square of the distances between sites and their assigned points in $X$. It is also shown in this paper how to compute the set of weights $W$ for a given set of capacities.

1. F. Aurenhammer, F. Hoffmann, and B. Aronov, Minkowski-type theorems and leastsquares partitioning, Proc. $8^{\text {th }}$ Ann. ACM Symp. on Computational Geometry (1992), 350-357.

A $k$-set of a finite set of points $S$ in $d$-dimensional space is a subset of $S$ of size $k$ that is all on one side of some hyperplane, while the other points in $S$ are all on the other side of the hyperplane. Since there is a one-to-one correspondence between $k$-sets in $d$-dimensional space and order $-k$ power cells in $(d-1)$-dimensional space, results about the number of 
$k$-sets of sets of points in $d$-dimensional space can be used for deriving results about the complexity of order $-k$ power diagrams in $(d-1)$-dimensional space. Let $S$ be a finite set in $d$-dimensional space. A $j$-set of $S$ is a $(\leq k)$-set of $S$ if $j \leq k$. Let $g_{d}(k, n)$ denote the maximum number of $(\leq k)$-sets of any set of $n$ points in $d$-dimensional space. In the following papers the computation of $g_{d}(k, n)$ is considered. In the first and second of the following papers it is shown, respectively, that $g_{2}(k, n) \leq 2 n k-2 k^{2}-k$ and $g_{2}(k, n)=k n$ for $2 k<n$. In the fourth paper it is shown that $g_{3}(k, n)=O\left(n^{2} k\right)$. In the fifth paper it is shown that $g_{3}(k, n)=O\left(n k^{5}\right)$. In the sixth paper it is shown that $g_{3}(k, n)=O\left(n k^{2} \log ^{8} n /(\log \log n)^{6}\right)$. Finally, in the seventh paper it is shown that $g_{d}(k, n)=\Theta\left(n^{\lfloor d / 2\rfloor} k^{\lceil d / 2\rceil}\right)$ as $n / k \rightarrow \infty$ for fixed $d$.

1. J. E. Goodman and R. Pollack, On the number of $k$-subsets of a set of $n$ points in the plane, J. of Combinatorial Theory, Series A 36 (1984), 101-104.

2. N. Alon and E. Györi, The number of small semispaces of a finite set of points in the plane, J. of Combinatorial Theory, Series A 41 (1986), 154-157.

3. E. Welzl, More on $k$-sets of finite sets in the plane, Discrete Comput Geom 1 (1986), 95-100.

4. R. Cole, M. Sharir, and C. K. Yap, On $k$-hulls and related problems, SIAM J. Comput. 16 (1987), 61-77.

5. B. Chazelle and F. P. Preparata, Halfspace range search: An algorithmic application of $k$-sets, Discrete Comput Geom 1 (1986), 83-93.

6. K. L. Clarkson, New applications of random sampling in computational geometry, Discrete Comput Geom 2 (1987), 195-222.

7. K. L. Clarkson and P. W. Shor, Applications of random sampling in computational geometry, II, Discrete Comput Geom 4 (1989), 387-421.

The second of the following papers presents a randomized algorithm for computing all levels of order 1 to $k, 1 \leq k \leq n$, in an arrangement of $n$ hyperplanes in $d$-space in expected time $O\left(k^{\lceil d / 2\rceil} n^{\lfloor d / 2\rfloor}\right)$ for $d \geq 4, O\left(k^{2} n \log n / k\right)$ for $d=3$, and $O(k n \log n / k)$ for $d=2$. From results in the first paper it follows that for fixed $d \geq 4$ these bounds are worst-case optimal as $n / k \rightarrow \infty$.

1. K. L. Clarkson and P. W. Shor, Applications of random sampling in computational geometry, II, Discrete Comput. Geom. 4 (1989), 387-421. 
2. K. Mulmuley, On levels in arrangements and Voronoi diagrams, Discrete Comput. Geom. 6 (1991), 307-338.

The following paper presents an algorithm that makes use of randomization in the data structure for the fast insertion or deletion of a hyperplane in an arrangement of hyperplanes in $d$-space, $d \leq 4$. For $d=2$ the insertion or deletion of a line in the arrangement with this algorithm requires expected $O\left(n^{2}\right)$ space and with high probability $O(n \log n)$ time and $O\left(n^{2} \log n\right)$ space. For $d=3$ the insertion or deletion of a hyperplane in the arrangement with this algorithm requires expected $O\left(n^{3}\right)$ space and with high probability $O\left(n^{2} \log n\right)$ time and $O\left(n^{3} \log n\right)$ space. Finally, for $d=4$ it requires with high probability $O\left(n^{3} \log n\right)$ time and $O\left(n^{4} \log n\right)$ space.

1. K. Mulmuley and S. Sen, Dynamic point location in arrangements of hyperplanes, Proc. $7^{\text {th }}$ Ann. ACM Symp. on Computational Geometry (1991), 132-141.

The following paper proposes a method for designing a correct finite precision algorithm for computing an arrangement of a set of lines in the plane. With this method infinite precision parameters that are close to the finite precision parameters for the lines are used without actually being represented.

1. V. J. Milenkovic, Verifiable implementations of geometric algorithms using finite precision arithmetic, Artificial Intelligence 37 (1988), 377-401.

In the following paper it is shown how to replace exact arithmetic with rounded arithmetic in an algorithm for computing line arrangements in the plane in such a way that the precision requirements of the algorithm are maintained while its complexity is reduced by a constant factor.

1. V. Milenkovic, Double precision geometry: A general technique for calculating line and segment intersections using rounded arithmetic, Proc. $30^{\text {th }}$ Ann. IEEE Symp. on the Foundations of Computer Science (1989), 500-505.

The following paper presents an analysis of the behavior of algorithms for computing line arrangements in the plane if the arithmetic operations involved make relative error $\epsilon$. Using $n$ lines in the plane as input, it is shown in this paper that two line arrangement algorithms, an $O\left(n^{2}\right)$ incremental algorithm and an $O\left(n^{2} \log n\right)$ sweepline algorithm, when implemented in a specific manner, will have $O(n \epsilon)$ relative error, i. e. will produce an arrangement of $n$ pseudolines each of which differs relatively from the corresponding line in the set of $n$ lines by at most $O(n \epsilon)$. It is also shown in this paper that an $O\left(n^{2} \log n\right)$ line arrangement algorithm exists which is perhaps of no practical interest but that has $O(\epsilon)$ relative error. 
1. S. Fortune and V. Milenkovic, Numerical stability of algorithms for line arrangements, Proc. $7^{\text {th }}$ Ann. ACM Symp. on Computational Geometry (1991), 334-341.

\section{Abstract Voronoi diagrams, discrete Voronoi diagrams, etc.}

In the following textbook a unifying approach to Voronoi diagrams in the plane is proposed through the introduction of abstract Voronoi diagrams. These diagrams are not based on sites and distance measures but on systems of bisecting curves. Given a finite index set $S$ it is assumed that for each pair of indices $p$ and $q$ in $S, p \neq q$, a bisecting curve $J(p, q)=J(q, p)$ exists which divides the plane into a $p$-region and a $q$-region. The Voronoi region for an index $p$ in $S$ is then defined as the intersection of all $p$-regions for different $q$ 's, and the abstract Voronoi diagram is accordingly defined as the union of the boundaries of the Voronoi regions for the indices in $S$. Assuming that the two regions separated by a bisecting curve are unbounded and that the Voronoi regions defined as above are path-connected and partition the plane, it is shown in this textbook that abstract Voronoi diagrams satisfy many of the properties satisfied by Voronoi diagrams that are based on the concept of distance, and that a class of metrics called 'nice' which includes many well-known metrics can be viewed in terms of the notion of abstract Voronoi diagrams. Finally, it is shown in this textbook how to merge two abstract Voronoi diagrams $V(L)$ and $V(R)$ for index sets $L$ and $R, L \cap R=\emptyset$, in a number of steps proportional to the cardinality of $L \cup R$ if the bisector of $L$ and $R$ contains no cycles, and how to compute in an $O(n \log n)$ divide-and-conquer fashion, relative to the complexity of elementary bisector operations, the Voronoi diagram for a set of $n$ sites based on a nice metric that admits acyclic partitions of the set of sites.

1. R. Klein, Concrete and abstract Voronoi diagrams, Springer-Verlag Lecture Notes in Computer Science 400 (1989).

The following paper presents an expected $O(n \log n)$ randomized algorithm for computing certain abstract Voronoi diagrams in the plane without the assumption about the existence of acyclic partitions.

1. K. Mehlhorn, C.O'Dunlaing, and S. Meiser, On the construction of abstract Voronoi diagrams, Springer-Verlag Lecture Notes in Computer Science 415 (1990), 227-239.

In the following paper the parallel computation of discrete Voronoi diagrams in the plane is considered. Given a positive integer $n$, a discrete universe $U$ is defined as the set of grid points in the plane of the form $p=\left(p_{x}, p_{y}\right), p_{x}, p_{y}$ integers, $1 \leq p_{x}, p_{y} \leq n$, and each point in $U$ is identified with a processor. Given a subset $S$ of $U$, to compute the discrete Voronoi 
diagram for $S$ means to store for every point $p$ in $U$ the coordinates of a point in $S$ closest to $p$ in a specified register of the processor assigned to $p$. In this paper algorithms are presented for computing in $O(\log n)$ time the discrete Voronoi diagram for $S$ based on the $L_{1}$ metric, and in $O\left(\log ^{3} n\right)$ time an approximation of the discrete Voronoi diagram for $S$ based on any $L_{k}$ metric, $1 \leq k \leq \infty$.

1. O. Schwarzkopf, Parallel computation of discrete Voronoi diagrams, Springer-Verlag Lecture Notes in Computer Science 349 (1989), 193-204.

In the first of the following papers Voronoi diagrams of point clusters are considered. For each cluster, i. e. a finite set of points in $d$-dimensional space, the distance from a point to the cluster is defined as the maximum Euclidean distance from the point to any point in the cluster. The Voronoi diagram of a finite set of clusters is then defined as the partition of the space into maximal domains, one for each cluster, such that a point belongs to the domain associated with a cluster if the point is closer to the cluster than it is to any other cluster. In this paper the close relationship between Voronoi diagrams of point clusters in $d$-dimensional space and the lower and upper envelopes of certain piecewise linear functions in $(d+1)$-dimensional space is demonstrated. It is also shown in this paper how this relationship can be exploited to compute the Voronoi diagram of a finite set of clusters in the plane in $O\left(n^{2} \alpha(n)\right)$ time, where $n$ is the sum of the cardinalities of the clusters and $\alpha(n)$ is the inverse of Ackermann's function. In particular, it is shown that if each cluster is of size one or two, then $O\left(n^{2}\right)$ time suffices and this is worst-case optimal. Finally, in the second paper a result about the complexity of the upper envelope of Voronoi surfaces in 3 -dimensional space is presented that implies that the complexity of the Voronoi diagram of $m$ clusters with a total of $n$ points in the plane is $O(m n \alpha(m n))$.

1. H. Edelsbrunner, L. J. Guibas, and M. Sharir, The upper envelope of piecewise linear functions: algorithms and applications, Discrete Comput. Geom. 4 (1989), 311-336.

2. D. P. Huttenlocher, K. Kedem, and M. Sharir, The upper envelope of Voronoi surfaces and its applications, Proc. $7^{\text {th }}$ Ann. ACM Symp. on Computational Geometry (1991), 194-203.

In the following two papers the peeper's Voronoi diagram for a set $S$ of sites in the plane is considered. Given a site $p$, the region of $p$ is the set of points in the plane for which $p$ is the closest site visible from each point in the set. Here visibility is constrained to a window, i. e. a line segment on a line that avoids the convex hull of $S$. The collection of regions of all sites is then the peeper's Voronoi diagram for $S$. In the second paper the relationship between peeper's Voronoi diagrams in the plane and upper envelopes in 3-dimensional space 
is demonstrated. It is also shown in this paper how this relationship can be exploited to compute the peeper's Voronoi diagram for a set of $n$ sites in worst-case optimal $O\left(n^{2}\right)$ time.

1. A. Baltsan and M. Sharir, On the shortest paths between two convex polyhedra, J. ACM 35 (1988), 267-287.

2. F. Aurenhammer and G. Stöckl, On the peeper's Voronoi diagram, SIGACT News 22 (1991), 50-59.

In the following paper dynamic Voronoi diagrams are considered. Given sets $P$ and $Q$ of $m$ and $n$ points in the plane, respectively, and assuming $Q$ is moving along a fixed direction, the problem considered is that of computing the Voronoi diagram for $P \cup Q$ at any time $t$. In this paper an $O(n m \log n m)$ algorithm is presented for processing the history of the Voronoi diagram for $P \cup Q$ as time passes. It is shown in this paper that this algorithm can be improved to require $O(m \log m+n \sqrt{m} \log n)$ time on average, and that $O(n m)$ space is required to store the history of the diagram in order to retrieve in $O(n+m)$ time the Voronoi diagram for $P \cup Q$ at any time $t$.

1. T. Tokuyama, Deformation of merged Voronoi diagrams with translation, Rep. TR870049, IBM Tokyo Research Laboratory (1988).

In the following paper an analysis is presented that can be used to obtain nontrivial bounds for the complexity of dynamic nearest and furthest point Voronoi diagrams in the plane. In particular it is observed in this paper that this analysis can be used to prove that $O\left(n^{3}\right)$ is a bound for the complexity of nearest and furthest Voronoi diagrams for $n$ points in the plane moving in different fixed directions at different constant velocities.

1. K. Imai, S. Sumino, and H. Imai, Minimax geometric fitting of two corresponding sets of points, Proc. $5^{\text {th }}$ Ann. ACM Symp. on Computational Geometry (1989), 266-275

In the following paper dynamic Voronoi diagrams are considered. Given a set of $n$ points in the plane that are moving, the problem considered is that of computing the Voronoi diagram for the set of points at any time $t$. In this paper preprocessing and query processing algorithms are presented for solving this problem. Assuming the points are in $k$-motion, i. e. their positions in the plane can be described in terms of polynomial functions of time with degree $\leq k$, and that it takes $O(k)$ time to find the roots of a polynomial with degree $O(k)$, it is shown in this paper that the preprocessing algorithm requires $O\left(k^{2} n^{3} \log n \cdot 2^{O\left(\alpha(n)^{5 k}\right)}\right.$ time and uses $O\left(k^{2} n^{3} 2^{O\left(\alpha(n)^{5 k}\right)}\right)$ space to store the preprocessing result, where $\alpha(n)$ is the inverse of Ackermann's function, and that the query processing algorithm is optimal, i. e. takes $O(n)$ time, at any time $t$. 
1. J.-J. Fu and R. C. T. Lee, Voronoi diagrams of moving points in the plane, International J. of Computational Geometry \& Applications 1 (1991), 23-32.

In the following paper dynamic Voronoi diagrams are considered. Given a set of $n$ points in the plane that are continuously moving along trajectories that satisfy certain assumptions, the problem considered is that of maintaining over time the Voronoi diagram for the set of points. In this paper a method for maintaining the Voronoi diagram over time in some useful data structure is presented and a numerically stable algorithm is given for the update over time of the topological structure of the diagram, using $O(\log n)$ worst-case optimal time per topological event. The main result in this paper is a proof that $O\left(n^{2} \lambda_{s}(n)\right)$ is an upper bound on the number of topological events, where $\lambda_{s}(n)$ is the maximum length of a DavenportSchinzel sequence on $n$ alphabets of order $s$, and $s$ is a constant depending on the motions of the points. In the special case where the points are moving at constant speed along straight lines, it is shown in this paper that this upper bound becomes $O\left(n^{3} 2^{\alpha(n)}\right)$, where $\alpha(n)$ is the inverse of Ackermann's function. Finally, it is shown in this paper that in the case where only $k$ of the $n$ points are moving, this bound becomes $O\left(k n \lambda_{s}(n)+(n-k)^{2} \lambda_{s}(k)\right)$ which is nearly quadratic for fixed $k$.

1. L. J. Guibas, J. S. B. Mitchell, and T. Roos, Voronoi diagrams of moving points in the plane, Graph-Theoretic Concepts in Computer Science, $17^{\text {th }}$ International Workshop, June 1991, Springer-Verlag Lecture Notes in Computer Science 570 (1992), 113-125.

In the first of the following papers $P$-Euclidean Voronoi diagrams in the plane are considered. Given polygons $P$ and $Q$ in the plane with $m$ and $n$ vertices, respectively, $P$ convex, and assuming $P$ is given together with a reference point $p$ in the interior of $P$, for a point $u$ in the interior of $Q, P(u)$ is said to denote the polygon obtained by translating $P$ so that the reference point $p$ coincides with $u$. The feasible region of $P$ inside $Q$ is defined as the set of points $u$ in the interior of $Q$ for which $P(u)$ is contained in $Q$. The feasible region is denoted by $F(P, Q)$ and may consist of several connected components, each of which is a polygon. Essentially, given edges $r$ and $s$ that are linear components of the boundaries of $P$ and $Q$, respectively, the Voronoi region for the pair $(r, s)$ is defined as the set of points $u$ in $F(P, Q)$ for which the minimum Euclidean distance between $P(u)$ and $Q$ is achieved by a point in $r$ and a point in $s$. Essentially, the $P$-Euclidean Voronoi diagram for $Q$ is defined as the planar skeleton formed by the boundaries of such Voronoi regions. In this paper it is observed that the boundary of $F(P, Q)$ has $O(m n)$ edges and can be computed in $O(m n \log m n)$ time by computing the Voronoi diagram for $Q$ based on the convex distance function defined with respect to $P$. It is also shown in this paper that the Euclidean Voronoi diagram for the boundary edges of $F(P, Q)$ and the $P$-Euclidean Voronoi diagram for $Q$ are 
identical in $F(P, Q)$ so that the $P$-Euclidean Voronoi diagram for $Q$ can be computed in $O(m n \log m n)$ time. In this paper dynamic $\tilde{P}(\theta)$-Euclidean Voronoi diagrams in the plane are also considered, where $\tilde{P}(\theta)$ denotes the rotation of the polygon $P$ around the reference point $p$ by an angle $\theta, 0 \leq \theta \leq 2 \pi$. In this paper it is shown that for $\theta$ changing from 0 to $2 \pi$ the $\tilde{P}(\theta)$-Euclidean Voronoi diagram for $Q$ can be computed in $O\left(m^{4} n \lambda_{16}(m n) \log m n\right)$ time, where $\lambda_{16}(m n)$ is the maximum length of Davenport-Schinzel sequences on $m n$ alphabets of order 16. Finally, the dynamic Voronoi diagram for $k n$-point rigid sets is considered in both of the following two papers. Assuming that the movement of each set is rigid and specified by a low-degree polynomial or well behaved rational functions of time, it is shown in the first paper that the complexity of the dynamic Voronoi diagram for the $k$ sets is $O\left(n^{2}\right)$ for $k=2,3$, and $O\left(n^{3} k^{4} \log ^{*} k\right)$ for $k \geq 4$. In the second paper the last complexity is improved to $O\left(n^{2} k^{2} \lambda_{s}(k)\right)$ for some fixed $s$.

1. H. Aonuma, H. Imai, K. Imai, and T. Tokuyama, Maximin location of convex objects in a polygon and related dynamic Voronoi diagrams, Proc. $6^{\text {th }}$ Ann. ACM Symp. on Computational Geometry (1990), 225-234.

2. D. P. Huttenlocher, K. Kedem, and J. M. Kleinberg, On dynamic Voronoi diagrams and the minimum Hausdorff distance for point sets under Euclidean motion in the plane, Proc. $8^{\text {th }}$ Ann. ACM Symp. on Computational Geometry (1992), 110-119.

In the following paper multiplicatively weighted crystal growth Voronoi diagrams are considered. These diagrams are essentially multiplicatively weighted Voronoi diagrams in which all regions are connected. They are obtained by allowing regions to grow simultaneously from their sites at their assigned growth rates while measuring the distance from a site to a point in the region of the site along a shortest path lying entirely within the region. In this paper an algorithm is presented for computing the crystal growth Voronoi diagram for $n$ sites. It is also shown in this paper that the optimal worst-case complexity of the diagram is $O\left(n^{2}\right)$ even though it is made up of $n$ connected regions.

1. B. F. Schaudt and R. L. Drysdale, Multiplicatively weighted crystal growth Voronoi diagrams, Proc. $7^{\text {th }}$ Ann. ACM Symp. on Computational Geometry (1991), 214-223.

\section{Delaunay triangulations and the Euclidean traveling salesman problem}

Solutions to Euclidean traveling salesman problems in the plane have been found to be related to Delaunay triangulations. The following paper presents an example of a set of points in the plane with a degenerate Delaunay triangulation that is not Hamiltonian. Essentially, a graph is Hamiltonian if it contains a cycle that passes through all of its nodes. 
1. V. Kantabutra, Traveling salesman cycles are not always subgraphs of Voronoi duals, Inform. Process. Lett. 16 (1983), 11-12.

The following paper presents an example of a set of points in the plane with a nondegenerate Hamiltonian Delaunay triangulation that does not contain a traveling salesman cycle. Essentially, a traveling salesman cycle for a set of points is a Hamiltonian cycle of minimum total length in the complete graph for the set.

1. M. B. Dillencourt, Traveling salesman cycles are not always subgraphs of Delaunay triangulations or of minimum weight triangulations, Inform. Process. Lett. 24 (1987), 339-342.

The following paper presents an example of a set of points in the plane with a nondegenerate Delaunay triangulation that is not Hamiltonian.

1. M. B. Dillencourt, A non-Hamiltionian, nondegenerate Delaunay triangulation, Inform. Process. Lett. 25 (1987), 149-151.

In the following paper it is shown that Delaunay triangulations are 1-tough. Essentially, a graph is 1 -tough if the removal of $k$ nodes and the arcs incident to these nodes leaves at most $k$ connected components. It is known that all Hamiltonian graphs are 1-tough. Thus, the result in the following paper seems to indicate that a Delaunay triangulation of a randomly generated set of points in the plane is almost certainly Hamiltonian.

1. M. B. Dillencourt, Toughness and Delaunay triangulations, Proc. $3^{\text {rd }}$ Ann. ACM Symp. on Computational Geometry (1987), 186-194.

In the following papers it is shown that in a certain sense Delaunay triangulations approximate complete Euclidean graphs. In the first paper it is shown that given a Delaunay triangulation defined with the $L_{1}$ metric, between any two vertices in the triangulation there is a path in the triangulation such that the ratio of the Euclidean length of this path to the Euclidean distance between the two vertices is bounded above by $\sqrt{10}$. In the second paper it is shown that if the Delaunay triangulation is defined with a convex distance function based on an equilateral triangle then the ratio is bounded above by 2 . In the third paper it is shown that if the Delaunay triangulation is defined with the Euclidean $\left(L_{2}\right)$ metric then the ratio is bounded above by $((1+\sqrt{5}) / 2) \pi \approx 5.08$. In the fourth paper the upper bound for the ratio is improved to $2 \pi / 3 \cos (\pi / 6) \approx 2.42$. Finally, in the first paper it is claimed that an example exists that shows that $\pi / 2$ is a lower bound for the ratio.

1. L. P. Chew, There is a planar graph almost as good as the complete graph, Proc. $2^{\text {nd }}$ Ann. ACM Symp. on Computational Geometry (1986), 169-177. 
2. L. P. Chew, There are planar graphs almost as good as the complete graph, J. of Computer and System Sciences 39 (1989), 205-219.

3. D. P. Dobkin, S. J. Friedman, and K. J. Supowit, Delaunay graphs are almost as good as complete graphs, Discrete Comput. Geom. 5 (1990), 399-407.

4. J. M. Keil and C. A. Gutwin, The Delaunay triangulation closely approximates the complete Euclidean graph, Springer-Verlag Lectures Notes in Computer Science 382 (1989), 47-56.

In the following paper it is shown how to obtain near-optimum heuristic solutions for large Euclidean traveling salesman problems in a short amount of time using Delaunay triangulations.

1. G. Reinelt, Fast heuristics for large geometric traveling salesman problems, ORSA J. on Computing 4 (1992), 206-217.

In the following paper experimental results are reported about the relation between solutions to Euclidean traveling salesman problems in the plane and Delaunay triangulations. These results show that the optimal tour for large Hamiltonian Delaunay triangulations averages about $0.1 \%$ above the optimal solution for the complete graph. They also show that regardless of its size, one can expect a Delaunay triangulation to contain on the average $99 \%$ of the arcs in the optimal solution for the complete graph.

1. W. R. Stewart, Euclidean traveling salesman problems and Voronoi diagrams, School of Business Administration, College of William and Mary, Manuscript (1992). 
